\title{
Symplectic Packings in Cotangent Bundles of Tori
}

\author{
F. Miller Maley, Jean Mastrangeli, and Lisa Traynor
}

\section{CONTENTS}

Introduction and Motivation

1. Tools and Main Results

2. Packing Capacities

3. Packing Analogues of Balls and Ellipsoids

4. Packing Analogues of Surface Products

5. Simplex Packing Computer Algorithms

Acknowledgements

References
Maley and Traynor received partial support from MSRI in the 1996-97 academic year. Traynor been partially supported by NSF grants DMS 9022140 and DMS 9971374.

Keywords: symplectic packings, symplectic capacities, lagrangian intersections, linear programming, Seshadri constants
Finding optimal packings of a symplectic manifold with symplectic embeddings of balls is a well known problem. In the following, an alternate symplectic packing problem is explored where the target and domains are $2 \mathrm{n}$-dimensional manifolds which have first homology group equal to $\mathbb{Z}^{\mathrm{n}}$ and the embeddings induce isomorphisms of first homology. When the target and domains are $\mathbb{T}^{\mathrm{n}} \times \mathrm{V}$ and $\mathbb{T}^{\mathrm{n}} \times \mathrm{U}$ in the cotangent bundle of the torus, all such symplectic packings give rise to packings of $\mathrm{V}$ by copies of $U$ under $\mathrm{GL}(\mathrm{n}, \mathbb{Z})$ and translations. For arbitrary dimensions, symplectic packing invariants are computed when packing a small number of objects. In dimensions 4 and 6 , computer algorithms are used to calculate the invariants associated to packing a larger number of objects. These alternate and classic symplectic packing invariants have interesting similarities and differences.

\section{INTRODUCTION AND MOTIVATION}

Some old and well known packing problems involve studying optimal arrangements of rigid copies of a standard shape. (See [Conway and Sloane 1988; Melissen 1997], for example.) In 1982, Gromov introduced problems regarding packing a symplectic manifold with the images of a standard shape under symplectomorphisms. In dimension 2, a symplectic manifold is simply a 2-dimensional manifold with an area form, and symplectomorphisms are precisely area preserving diffeomorphisms. In higher dimensions, a symplectic manifold is a smooth $2 n$ dimensional manifold equipped with a closed 2-form $\omega$ which is nondegenerate in the sense that $\omega^{n}$ is a volume form. Even-dimensional euclidean space and the cotangent bundle of the torus, denoted $T^{*} \mathbb{T}^{n}$, have canonical symplectic structures and will be the main symplectic manifolds studied in this paper. In higher dimensions, symplectic diffeomorphisms still preserve volume, but an exact understanding of symplectic diffeomorphisms is elusive. Results about symplectic packings demonstrate that sym- 
plectic diffeomorphisms are sometimes quite flexible while at other times are extremely rigid.

Gromov defined an invariant of a symplectic manifold by studying a symplectic embedding of a ball into the symplectic manifold. Let $\omega_{0}$ denote the standard symplectic form on $\mathbb{R}^{2 n}$. The Gromov width of a symplectic manifold $(M, \omega)$ is

$$
\begin{aligned}
g(M, \omega)=\sup \left\{\pi r^{2} \mid\right. & \psi^{*} \omega=\omega_{0} \text { for some } \\
& \text { embedding } \left.\psi: B^{2 n}(r) \rightarrow M\right\} .
\end{aligned}
$$

As an illustration of the nontriviality of this invariant, Gromov showed that the infinite volume cylinder

$$
Z^{2 n}(1):=\left\{x_{1}^{2}+y_{1}^{2}<1\right\} \times \mathbb{R}^{2 n-2}
$$

has $g\left(Z^{2 n}(1), \omega_{0}\right)=\pi$. This is often referred to as "Gromov's Nonsqueezing Theorem"; see Theorem 2.3. The width invariant can be generalized to the ball k-packing width (or ball k-width) by studying $k$ symplectic embeddings of a ball with disjoint images:

(0.1) $g_{k}(M, \omega)=\sup \left\{\pi r^{2} \mid\right.$ there are $k$ embeddings of $\left(B^{2 n}(r), \omega_{0}\right)$ into $(M, \omega)$ with pairwise disjoint images $\}$.

Section 2 describes how these ball packing widths satisfy properties that qualify them to be symplectic capacities. Although these invariants are quite easy to define, they are extremely difficult to calculate. In some cases, such as $\mathbb{C P}^{2}$ or $S^{2} \times S^{2}$, the ball packing widths are known for an arbitary number of balls. These calculations were initiated in [Gromov 1985] as applications of his theory of pseudoholomorphic curves, extended in [McDuff and Polterovich 1994] using more developed ideas of algebraic geometry, and further extended in [Biran 1997; 1999b], by incorporating results from the framework of Taubes' theory of Gromov invariants.

In this paper, a new symplectic invariant is defined and computed for symplectic manifolds that satisfy a special topological condition. Instead of considering all symplectic manifolds, we restrict attention to symplectic manifolds with first homology equal to $\mathbb{Z}^{n}$, where $2 n$ is the dimension of the manifold:

$$
\mathcal{M}_{\mathbb{Z}^{n}}:=\left\{\left(M^{2 n}, \omega\right) \mid H_{1}(M ; \mathbb{Z}) \simeq \mathbb{Z}^{n}\right\} .
$$

If $U$ is an open, contractible subset of $\mathbb{R}^{n}$ then the subspace $T^{n} \times U$ of $T^{*} \mathbb{T}^{n}$ is an element of $\mathcal{M}_{\mathbb{Z}^{n}}$.
Throughout this paper, $\triangle^{n}(r)$ will denote the interior of the $n$-dimensional simplex with the $n+1$ vertices $(0, \ldots, 0),(r, 0, \ldots, 0), \ldots,(0, \ldots, 0, r)$, and $P^{2 n}(r)$ will denote the corresponding subset of $T^{*} \mathbb{T}^{n}$ :

$$
P^{2 n}(r):=\mathbb{T}^{n} \times \triangle^{n}(r)
$$

$P^{2 n}(r)$ can be thought of as an $\mathcal{M}_{\mathbb{Z}^{n}}$ counterpart of $B^{2 n}(\sqrt{r})$ since for all $\varepsilon>0$, there exist symplectic embeddings

$$
B^{2 n}(\sqrt{r-\varepsilon}) \rightarrow P^{2 n}(r) \rightarrow B^{2 n}(\sqrt{r}) ;
$$

see [Traynor 1995], for example. Thus embeddings of $B^{2 n}(\sqrt{r})$ into a symplectic manifold give rise to embeddings of $P^{2 n}(r)$, and embeddings of $P^{2 n}(r)$ give rise to embeddings of $B^{2 n}(\sqrt{r-\varepsilon})$ for any $\varepsilon>$ 0 . Thus there is no quantitative difference between looking at symplectic packings with $B^{2 n}(\sqrt{r})$ and with $P^{2 n}(r)$. But when restricting to symplectic manifolds in $\mathcal{M}_{\mathbb{Z}^{n}}$, it is possible to add the condition that the symplectic embeddings of $P^{2 n}(r)$ induce an isomorphism on the level of first homology. The simplex k-packing width (or simplex $k$-width) $s_{k}: \bigcup_{n} \mathcal{M}_{\mathbb{Z}^{n}} \rightarrow[0, \infty]$, is defined by

(0.3) $s_{k}(M, \omega)=\sup \{\pi r \mid$ there are $k$ embeddings of $\left(P^{2 n}(r), \omega_{0}\right)$ into $(M, \omega)$ with pairwise disjoint images and inducing isomorphisms $\left.H_{1}(P(r)) \stackrel{\simeq}{\longrightarrow} H_{1}(M)\right\}$.

It is easy to check that these are symplectic invariants. Section 2 shows that these invariants satisfy a set of axioms analogous to the capacity axioms.

Thus for a symplectic manifold that satisfies the nontriviality condition on first homology, one has both the ball packing and simplex packing widths, and it is easy to see that

$$
s_{k}(M, \omega) \leq g_{k}(M, \omega) \leq \nu_{k}(M, \omega)
$$

where $\nu_{k}$ is the upper bound given by the fact that symplectic embeddings must preserve volume. The natural question arises: Is $s_{k}$ different from $g_{k}$ ?

The aim of this paper is to highlight some interesting similarities and differences between these two families of packing invariants. In the process of calculating simplex widths, connections between convex geometry, computer algorithms, and symplectic topology are illustrated. 


\section{TOOLS AND MAIN RESULTS}

Whereas algebraic geometry is a crucial tool to calculate ball packing widths, the main tool to calculate the simplex packing widths is the following theorem.

Theorem 1.1 (= Corollary 2.6). Suppose $V$ is an open, connected subset of $\mathbb{R}^{n}$ with $H_{1}(V ; \mathbb{Z})=0$. Then

$s_{k}\left(\mathbb{T}^{n} \times V, \omega_{0}\right)=\sup \{\pi r \mid$ there are $k$ integral affine transformations taking $\triangle(r)$ into $V$ and having pairwise disjoint images $\}$.

By an integral affine transformation we mean the composition of an element of $\mathrm{GL}(n, \mathbb{Z})$ with a translation in $\mathbb{R}^{n}$. It is easy to see that, given $k$ transformations that satisfy the conditions of the theorem, one can construct a symplectic packing that satisfies the nontriviality condition on first homology (see Remark 2.7). The nontrivial implication is that to every packing of $\mathbb{T}^{n} \times V$ by symplectic images of $P^{2 n}(r)=\mathbb{T}^{n} \times \triangle^{n}(r)$, one associates a packing of $V$ by copies of $\triangle^{n}(r)$ under $\operatorname{GL}(n, \mathbb{Z})$ and translations. In particular, this reduces a packing problem of some 4-dimensional manifolds to packing a 2-dimensional subset of $\mathbb{R}^{2}$ with the images of a right, isosceles triangle under $\mathrm{GL}(2, \mathbb{Z})$ and translations. Whereas the pseudo-holomorphic technique is most powerful in dimension 4, this theorem applies equally to arbitrary dimensions. The proof of this theorem can be viewed as an extension of a rigidity result from [Sikorav 1989], and is closely connected to the rigidity of exact lagrangian submanifolds. In fact, when $n=2$, a version of Theorem 1.1 holds with weaker conditions on $V$. For example, an analogous result holds when $V$ is a punctured disc and $s_{k}$ is defined by considering symplectic packings with $\left(\psi_{i}\right)_{*}$ injective for all $i$. This is a consequence of Giroux's results [1994] about incompressible lagrangian tori in $\mathbb{T}^{2} \times\left(\mathbb{R}^{2} \backslash\{0\}\right)$. See Remark 2.14. It would be interesting to understand more examples where analogues of Theorem 1.1 hold.

Interesting similarities and differences between the ball packing and simplex packing widths are illustrated by the following result, to be proved later.

Theorem 1.2. Let $P=P^{4}(1)=\mathbb{T}^{2} \times \triangle(1) \subset T^{*} \mathbb{T}^{2}$. These are the ball $k$-widths $g_{k}(P)$ and simplex $k$ widths $s_{k}(P)$, for $1 \leq k \leq 9$ :

\begin{tabular}{|c|ccccccccc|}
\hline$k$ & 1 & 2 & 3 & 4 & 5 & 6 & 7 & 8 & 9 \\
\hline$g_{k}(P) / \pi$ & 1 & $\frac{1}{2}$ & $\frac{1}{2}$ & $\frac{1}{2}$ & $\frac{2}{5}$ & $\frac{2}{5}$ & $\frac{3}{8}$ & $\frac{6}{17}$ & $\frac{1}{3}$ \\
$s_{k}(P) / \pi$ & 1 & $\frac{1}{2}$ & $\frac{1}{2}$ & $\frac{1}{2}$ & $\frac{2}{5}$ & $\frac{6}{17}$ & $\frac{1}{3}$ & $\frac{1}{3}$ & $\frac{1}{3}$ \\
\hline
\end{tabular}

Remark 1.3. It is interesting that $\frac{6}{17}$ appears in both cases but for packing different numbers of objects. This phenomenon will continue to appear in upcoming calculations. It is work in progress by Traynor to understand why this happens.

The ball packing width calculations in Theorem 1.2 follow from ball packing width calculations for $\mathbb{C P}^{2}$ done in [Gromov 1985] and [McDuff and Polterovich 1994]. The simplex width calculations can easily be done directly when $k \leq 5$ using techniques described in Sections 3 and 4 . For larger $k, s_{k}$ has been calculated by the computer program described in Section 5. Some optimal ball and simplex packings can be visualized as in Figure 1.4. Explicit ball packing constructions are unknown for nonsquares beyond 6 .

In fact, $g_{k}\left(P^{4}(1)\right)$ is known for all values of $k$. Biran [1997] proved that $g_{k}\left(P^{4}(1)\right)=\pi / \sqrt{k}$ for all $k \geq 9$. Equivalently, it is possible to come arbitrarily close to exhausting all the volume of $P^{4}(1)$ by the image of symplectic embeddings of $k$ balls when $k \geq 9$. When $k$ is a square, one can similarly get

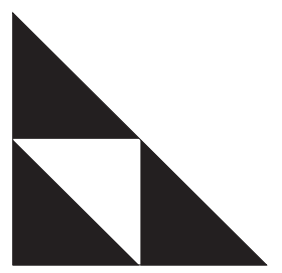

(a)

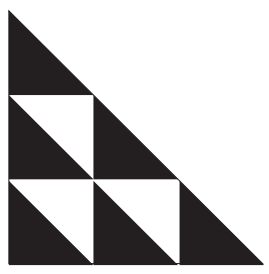

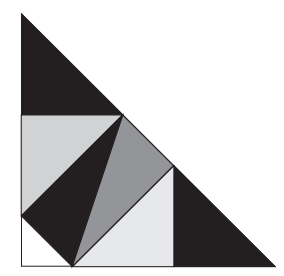

(b)

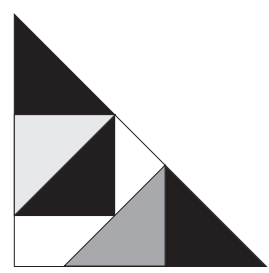

(c)

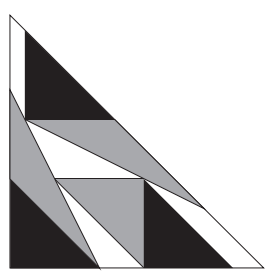

FIGURE 1.4. (a) Optimal ball or simplex packings with $k \leq 4$ and $k=9$ objects. (b) Optimal ball packings with $k=6$ balls [Traynor 1995]. (c) Optimal simplex packings with $k=5,6$ simplices. 
"full" simplex packings. However, in contrast to $g_{k}$, the value of $s_{k}$ will always be rational (see Remark 5.4). Although $g_{k}$ and $s_{k}$ differ radically for $k \geq 9$ and not a perfect square, there are some interesting similarities between lower bound estimates for $s_{k}$ and a version of Seshadri constants $h_{k}$ from algebraic geometry. Namely, consider

$h_{k}(M, \omega)=\sup \left\{\pi r^{2} \mid\right.$ the cohomology class $\Theta^{*}[\omega]-\pi r^{2} \sum_{i=1}^{k} e_{i}$ admits a Kähler representative on the blow-up of $M$ at $k$ (generic) points\}.

Here $\Theta: M^{\prime} \rightarrow M$ is the blow-up of $M$ at $k$ points, and $e_{i}$ denotes the Poincaré dual to the exceptional divisor over the $i$-th blown-up point. Similar to $s_{k}$, $h_{k}$ is bounded above by $g_{k}$ :

$$
h_{k}\left(\mathbb{C} \mathbb{P}^{2}\right) \leq g_{k}\left(\mathbb{C} \mathbb{P}^{2}\right)=g_{k}\left(B^{4}(1)\right) .
$$

A conjecture in [Nagata 1959] leads to the conjecture that $h_{k}\left(\mathbb{C P}^{2}\right)=g_{k}\left(\mathbb{C P}^{2}\right)=g_{k}\left(P^{4}(1)\right)$, for all $k$. See [McDuff and Polterovich 1994]. Although Nagata's conjecture is still open, a number of people have made estimates for $h_{k}\left(\mathbb{C P}^{2}\right)$.

Theorem 1.5. (a) $[\mathrm{Xu} 1994]$ For all $j \in \mathbb{N}$,

$$
h_{j}\left(\mathbb{C P}^{2}\right) \geq \frac{\sqrt{j-1}}{j} \pi .
$$

(b) [Biran 1999a] For all $k \in \mathbb{N}$,

$h_{k^{2}+1}\left(\mathbb{C P}^{2}\right) \geq \frac{2 k}{2 k^{2}+1} \pi$ and $h_{k^{2}+2}\left(\mathbb{C P}^{2}\right) \geq \frac{k}{k^{2}+1} \pi$.

Notice that Biran's calculations for $h_{j}$, when $j$ is one or two larger than a square, are stronger than $\mathrm{Xu}$ 's general estimate for $h_{j}$. When $j$ is one larger than a square, the following estimates of $s_{j}$, proved in Section 3, agree with Xu's estimates of $h_{j}$ and with Biran's estimate of $h_{j+1}$. When $j=k^{2}+2$ for $k \geq 4$, our estimate of $s_{j}$ is greater than $\mathrm{Xu}$ 's estimate of $h_{j}$.

Theorem 1.6 (= Theorem 3.1). For $k \geq 2$, we have

$$
\begin{aligned}
& s_{k^{2}+1}\left(P^{4}(1)\right) \geq \frac{k}{k^{2}+1} \pi, \\
& s_{k^{2}+2}\left(P^{4}(1)\right) \geq \frac{k-1}{k^{2}-k+1} \pi .
\end{aligned}
$$
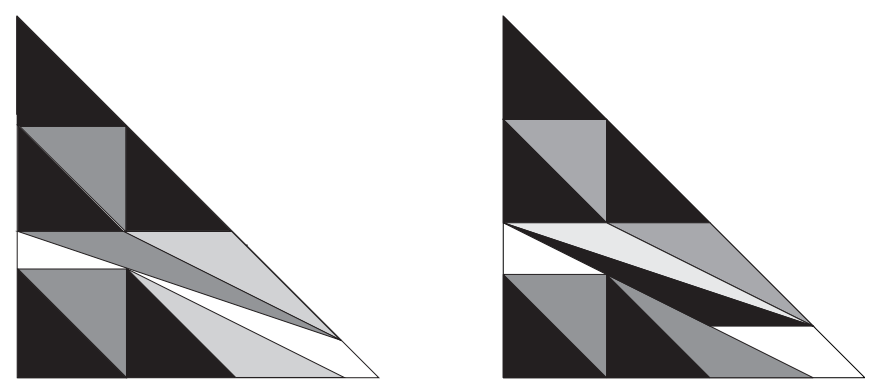

FIGURE 1.7. Left: a $k^{2}+1=10$ simplex packing. Right: a $k^{2}+2=11$ simplex packing.

The following Theorem and Conjecture gives Biran's exact calculations of $g_{k}$ from [Biran 1997] and known and conjectured lower bound estimates on $h_{k}$ made in [Biran 1999a]. These estimates are obtained from continued fraction expansions of $\sqrt{k}$ which lead to minimal solutions to Pell's equation. The calculations and estimates on $s_{k}$ are again done by the computer program described in Section 5.

\section{Theorem and Conjecture 1.8. Let}

$$
P=P^{4}(1)=\mathbb{T}^{2} \times \triangle(1) \subset T^{*} \mathbb{T}^{2} .
$$

Table 1 gives the exact ball $k$-widths $g_{k}(P)$, the exact calculations of the simplex widths $s_{10}, s_{11}, s_{12}$, and estimates and conjectures on lower bounds for the

\begin{tabular}{|c|c|c|c|c|c|c|c|c|c|c|c|c|c|c|}
\hline$k$ & 10 & 12 & 12 & 13 & 14 & 15 & 16 & 17 & 18 & 19 & 20 & 21 & 22 & 23 \\
\hline$g_{k}(P) / \pi$ & $\frac{1}{\sqrt{10}}$ & $\frac{1}{\sqrt{11}}$ & $\frac{1}{\sqrt{12}}$ & $\frac{1}{\sqrt{13}}$ & $\frac{1}{\sqrt{14}}$ & $\frac{1}{\sqrt{15}}$ & $\frac{1}{4}$ & $\frac{1}{\sqrt{17}}$ & $\frac{1}{\sqrt{18}}$ & $\frac{1}{\sqrt{19}}$ & $\frac{1}{\sqrt{20}}$ & $\frac{1}{\sqrt{21}}$ & $\frac{1}{\sqrt{22}}$ & $\frac{1}{\sqrt{23}}$ \\
\hline$h_{k}\left(\mathbb{C P}^{2}\right) / \pi$ & $\geq \frac{6}{19}$ & $\geq \frac{3}{10}$ & $\geq \frac{2}{7}$ & $\geq \frac{180}{649}$ & $\stackrel{?}{\geq} \frac{4}{15}$ & $\geq \frac{1}{4}$ & $\frac{1}{4}$ & $\stackrel{?}{\geq} \frac{8}{33}$ & $\stackrel{?}{\geq} \frac{4}{17}$ & $\stackrel{?}{\geq} \frac{39}{170}$ & $\geq \frac{2}{9}$ & $\stackrel{?}{\geq} \frac{12}{55}$ & $\stackrel{?}{\geq} \frac{42}{197}$ & $\stackrel{?}{\geq} \frac{5}{24}$ \\
\hline$s_{k}(P) / \pi$ & $\frac{3}{10}$ & $\frac{2}{7}$ & $\frac{15}{56}$ & $\stackrel{!}{\geq} \frac{6}{23}$ & $\stackrel{!}{\geq} \frac{20}{79}$ & $\stackrel{!}{\geq} \frac{1}{4}$ & $\frac{1}{4}$ & $\stackrel{!}{\geq} \frac{4}{17}$ & $\stackrel{!}{\geq} \frac{3}{13}$ & $\stackrel{!}{\geq} \frac{2}{9}$ & $\stackrel{!}{\geq} \frac{21}{97}$ & $\stackrel{!}{\geq} \frac{4}{19}$ & $\stackrel{!}{\geq} \frac{7}{34}$ & $\geq \frac{21}{104}$ \\
\hline
\end{tabular}
remaining $s_{k}$ and the Seshadri constants $h_{k}$.

For arbitrary dimensions, using holomorphic curve techniques, Gromov proved that $g_{k}\left(\mathbb{C P}^{n}\right) \leq \frac{\pi}{2}$ when $1<k \leq 2^{n}$. This implies that $g_{k}\left(P^{n}(1)\right) \leq \frac{\pi}{2}$ for

TABLE 1. Results for Theorem 1.8. $\geq$ denotes a known lower bound, $\stackrel{?}{\geq}$ denotes a conjectured lower bound, and $\stackrel{!}{\geq}$ denotes a known lower bound, conjectured optimal. 
these values of $k$, and thus $s_{k}\left(P^{n}(1)\right) \leq \frac{\pi}{2}$. However, a direct, more elementary proof is given in Section 3 for this simplex widths' upper bound.

\section{Theorem 1.9 (= Theorem 3.2).}

$$
s_{k}\left(P^{2 n}(1)\right) \leq \pi / 2 \quad \text { for } 1<k \leq 2^{n} .
$$

Equality holds for $1<k \leq n+1$.

Remark 1.10. As a complement to Gromov's obstruction calculations for $\mathbb{C P}^{n}$, McDuff and Polterovich proved, by techniques of algebraic geometry, that there is a full ball $2^{n}$-packing of $\mathbb{C P}^{n}$, and thus $g_{k}\left(P^{2 n}(1)\right)=\pi / 2$ for $1<k \leq 2^{n}$. This can alternatively be proved using the ball packing constructions from [Traynor 1995]. The fact that the simplex packing widths can be precisely calculated for $1<k \leq n+1$ follows from explicit constructions. In fact, it is known that equality holds for more values of $k$, but it is easy to prove for $1<k \leq n+1$. Moreover, for $n=3,4$, it is proved in [Mastrangeli 1997] that $s_{k}\left(P^{2 n}(1)\right)=\pi / 2$ for $1<k \leq 2^{n}$, and it is still work in progress by Mastrangeli to verify that this statement holds for arbitrary dimensions.

Next, consider a variation of $P^{2 n}(r)$ :

$$
P\left(\alpha_{1}, \ldots, \alpha_{n}\right):=\mathbb{T}^{n} \times \triangle\left(\alpha_{1}, \ldots, \alpha_{n}\right),
$$

where $\triangle\left(\alpha_{1}, \ldots, \alpha_{n}\right)$ is the simplex with the $(n+1)$ vertices $(0, \ldots, 0),\left(\alpha_{1}, 0, \ldots, 0\right), \ldots,\left(0, \ldots, 0, \alpha_{n}\right)$. Just as $P^{n}(r)$ can be thought of as an analogue of a ball of radius $\sqrt{r}$, so $P\left(\alpha_{1}, \ldots, \alpha_{n}\right)$ is an $\mathcal{M}_{\mathbb{Z}^{n-}}$ analogue of the ellipsoid

$$
\begin{aligned}
E\left(\sqrt{\alpha_{1}}, \ldots, \sqrt{\alpha_{n}}\right) & \\
& :=\left\{\frac{1}{\alpha_{1}}\left(x_{1}^{2}+y_{1}^{2}\right)+\cdots+\frac{1}{\alpha_{n}}\left(x_{n}^{2}+y_{n}^{2}\right)<1\right\} .
\end{aligned}
$$

Gromov's Nonsqueezing Theorem implies that $g_{1}\left(E\left(\sqrt{\alpha_{1}}, \ldots, \sqrt{\alpha_{n}}\right)\right)=\pi \alpha_{1}$ when $\alpha_{1} \leq \cdots \leq \alpha_{n}$.

However, higher widths have not been previously calculated, because most of the ellipsoids do not have nice algebrogeometric counterparts. At best, for some choices of $\alpha_{i}$, the ellipsoids correspond to orbifolds of weighted projective spaces, but the holomorphic curve theory for orbifolds has not yet been developed. Although the ball packing widths are not known, in Section 3 some "convexity" arguments give rise to the following simplex width calculations.
Theorem 1.11 (= Theorem 3.10). If $\alpha_{1} \leq \cdots \leq \alpha_{n}$ then

$$
s_{1}\left(P\left(\alpha_{1}, \ldots, \alpha_{n}\right)\right)=\alpha_{1} \pi,
$$

and

$$
s_{2}\left(P\left(\alpha_{1}, \ldots, \alpha_{n}\right)\right)=\min \left\{\alpha_{1}, \frac{1}{2} \alpha_{n}\right\} \pi .
$$

As a corollary, it is possible to calculate the second ball packing width of an ellipsoid:

Corollary 1.12 (= Corollary 3.11). If $\alpha_{1} \leq \cdots \leq \alpha_{n}$ then

$$
g_{2}\left(E\left(\sqrt{\alpha_{1}}, \ldots, \sqrt{\alpha_{n}}\right)\right)=\min \left\{\alpha_{1}, \frac{1}{2} \alpha_{n}\right\} \pi .
$$

In Section 4 , an analogue of the polydisc $D^{2} \times D^{2}$ is studied. Again there are interesting similarities and differences between the ball and simplex packing widths. Let $\square(1,1) \subset \mathbb{R}^{2}$ be the interior of the square with vertices $(0,0),(1,0),(0,1),(1,1)$ and $\Sigma^{4}(1):=\mathbb{T}^{2} \times \square(1,1)$. The following calculations of $g_{k}$ are consequences of Biran's calculations [1997] of $g_{k}\left(S^{2} \times S^{2}\right)$. The calculations of $s_{k}$ for $k=1,2,3$ are done in Section 4 while for higher $k$ they are done by the computer program described in Section 5 .

Theorem 1.13. For $\Sigma=\mathbb{T}^{2} \times \square(1,1)$, the ball packing widths and simplex packing widths are:

\begin{tabular}{|c|cccccccc|}
\hline$k$ & 1 & 2 & 3 & 4 & 5 & 6 & 7 & 8 \\
\hline$g_{k}(\Sigma) / \pi$ & 1 & 1 & $\frac{2}{3}$ & $\frac{2}{3}$ & $\frac{3}{5}$ & $\frac{4}{7}$ & $\frac{8}{15}$ & $\frac{1}{2}$ \\
$s_{k}(\Sigma) / \pi$ & 1 & 1 & $\frac{2}{3}$ & $\frac{4}{7}$ & $\frac{1}{2}$ & $\frac{1}{2}$ & $\frac{1}{2}$ & $\frac{1}{2}$ \\
\hline
\end{tabular}
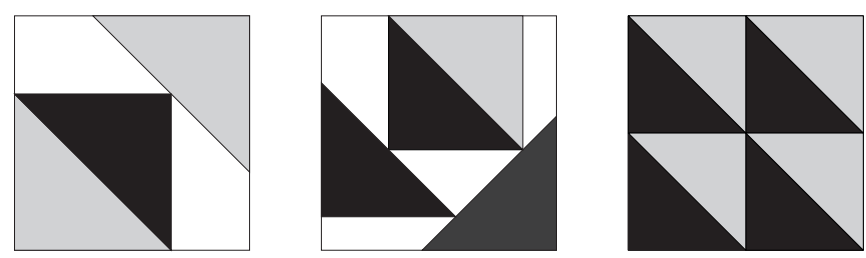

FIGURE 1.14. Optimal simplex packings with 3,4 , and 8 simplices.

Biran [1997] proved that, for $k \geq 8$, there exists a full packing of $S^{2} \times S^{2}$. Equivalently, $g_{k}\left(\Sigma^{4}(1)\right)=$ $\sqrt{2 / k} \pi$, for $k \geq 8$. In contrast, the computer algorithm implies that $s_{k}$ will always be rational; see Remark 5.4. Alternatively, the following theorem demonstrates that $g_{k}$ and $s_{k}$ often differ.

Theorem 1.15. When $k$ is odd, $\Sigma^{4}(1)$ does not admit a full simplex packing:

$$
s_{k}\left(\Sigma^{4}(1)\right)<g_{k}\left(\Sigma^{4}(1)\right), \quad \text { for } k \geq 9 \text { and odd. }
$$


Proof. By Theorem 1.1, full packings of $\Sigma^{4}(1)$ exist only if there exist full packings of $\square^{2}(1)$ with images of $\triangle^{2}(r)$ under $\operatorname{GL}(2, \mathbb{Z})$ and translations. The result then follows immediately from a result that states a square can be partitioned into $k$ triangles of equal area only when $k$ is even. See, for example, the argument in [Stein and Szabó 1994].

More precisely:

Theorem 1.16. Let $\Sigma=\mathbb{T}^{2} \times \square(1,1)$. Table 2 gives the known optimal, known and conjectured optimal, and known lower bounds for the simple $k$-widths $s_{k}(\Sigma)$.

In higher dimensions, let $\square\left(\alpha_{1}, \ldots, \alpha_{n}\right) \subset \mathbb{R}^{n}$ denote the open box

$$
\square\left(\alpha_{1}, \ldots, \alpha_{n}\right):=\left\{\left(y_{1}, \ldots, y_{n}\right) \mid 0<y_{i}<\alpha_{i}\right\} .
$$

Then $\Sigma\left(\alpha_{1}, \ldots, \alpha_{n}\right):=\mathbb{T}^{n} \times \square\left(\alpha_{1}, \ldots, \alpha_{n}\right)$ is the analogue of the product of the 2-dimensional discs of radius $\sqrt{\alpha_{1}}, \ldots, \sqrt{\alpha_{n}}$ since, as shown in [Traynor 1995], for all $\varepsilon>0$ there exist symplectic embeddings

$D^{2}\left(\sqrt{\alpha_{1}-\varepsilon}\right) \times \cdots \times D^{2}\left(\sqrt{\alpha_{n}-\varepsilon}\right) \rightarrow \Sigma\left(\alpha_{1}, \ldots, \alpha_{n}\right)$

and

$$
\Sigma\left(\alpha_{1}, \ldots, \alpha_{n}\right) \rightarrow D^{2}\left(\sqrt{\alpha_{1}}\right) \times \cdots \times D^{2}\left(\sqrt{\alpha_{n}}\right) .
$$

The convexity arguments in Section 3 give rise to the following two results.

Theorem 1.17 (= Theorem 4.1). If $\alpha_{1} \leq \cdots \leq \alpha_{n}$ then $s_{k}\left(\Sigma\left(\alpha_{1}, \ldots, \alpha_{n}\right)\right)=\alpha_{1} \pi$ for $1 \leq k \leq n$ !.

Theorem 1.18 (= Theorem 4.2). If $\alpha_{1} \leq \alpha_{2}$ then

$$
s_{3}\left(\Sigma\left(\alpha_{1}, \alpha_{2}\right)\right)=\min \left\{\alpha_{1}, \frac{1}{3}\left(\alpha_{1}+\alpha_{2}\right)\right\} \pi .
$$

The proof of Theorem 1.17 is fairly easy, but that of Theorem 1.18 illustrates the difficulty of doing simplex width calculations directly. The following conjecture has been tested using the computer program described in Section 5.
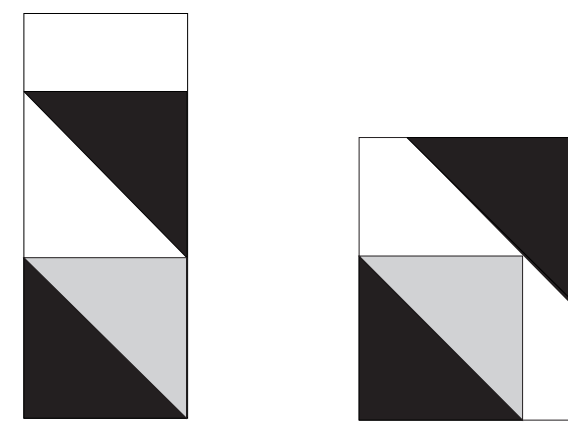

FIGURE 1.19. Optimal simplex packing configurations with $k=3$ objects when $\alpha_{2} \geq 2 \alpha_{1}$ and when $\alpha_{2} \leq 2 \alpha_{1}$.

Conjecture 1.20. For $\alpha_{1} \leq \alpha_{2}$, $s_{4}\left(\Sigma\left(\alpha_{1}, \alpha_{2}\right)\right)=\min \left\{\alpha_{1}, \max \left\{\frac{1}{2} \alpha_{2}, \frac{2}{7}\left(\alpha_{1}+\alpha_{2}\right)\right\}\right\} \pi$.

For higher dimensions, by results from [McDuff and Polterovich 1994], it is known that there is a full ball $k$-packing of the 6 -dimensional polydisc and thus of $\Sigma(1,1,1)$ for $k=3 ! j^{3}$, where $j \in \mathbb{N}$. As described in Section 5, computer calculations lead to some precise values for $s_{k}$ and thus to estimates of $g_{k}$.

Theorem 1.21. Let $\Sigma^{3}(1)=T^{3} \times \square(1,1,1)$. Then

$$
s_{7}\left(\Sigma^{3}(1)\right)=\frac{4}{5} \pi \quad \text { and } \quad s_{8}\left(\Sigma^{3}(1)\right)=\frac{3}{4} \pi .
$$

As a consequence,

$g_{7}\left(S^{2} \times S^{2} \times S^{2}\right) \geq \frac{4}{5} \pi \quad$ and $\quad g_{8}\left(S^{2} \times S^{2} \times S^{2}\right) \geq \frac{3}{4} \pi$.

There are many interesting questions beyond calculating the simplex width capacities of a fixed symplectic manifold. For example, it would be interesting to understand the possible effect of removing subsets of volume 0 on the simplex widths. Analogous but harder results were obtained for the HoferZehnder capacities in [Tokieda 1996; 1997]. Section 4 gives some results and conjectures about the possible beginnings of the "simplex capacity spec-

\begin{tabular}{|c|c|c|c|c|c|c|c|c|c|c|c|c|c|c|c|c|c|}
\hline$k$ & $\begin{array}{lllll}9 & 10 & 11 & 12 & 13\end{array}$ & 14 & 15 & 16 & $17 \quad 18$ & 19 & 20 & 21 & 22 & 23 & 24 & 25 & 26 & 27 & 28 & 29 & 30 \\
\hline$\frac{s_{k}(\Sigma)}{\pi}$ & 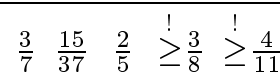 & $=\overline{2}$ & $\geq \frac{15}{44}$ & 117 & \begin{tabular}{ll}
$!$ \\
\hdashline
\end{tabular} & $\geq \frac{5}{16}$ & $\geq \frac{11}{36}$ & $\geq \frac{1}{4}$ & $\geq \frac{7}{24}$ & & $\geq \frac{5}{18}$ & & & $\geq \frac{43}{164}$ & $\geq \frac{8}{31}$ & $\geq \frac{16}{63}$ & $\geq \frac{52}{207}$ \\
\hline
\end{tabular}
trum", the sequence of numbers that can be realized as capacities for $\mathbb{T}^{2} \times U$ where $U$ is an open subset of $\square(1)$.

TABLE 2. Bounds for $\Sigma=\mathbb{T}^{2} \times \square(1,1)$ (Theorem 1.16). $\geq$ denotes a known lower bound, and $\stackrel{!}{\geq}$ denotes a known lower bound, conjectured optimal. 
Conjecture 1.22 (= Conjecture 4.5). Consider an open, contractible set $U \subset \square(1)$. Let $s_{1}=r_{1} \pi, s_{2}=$ $r_{2} \pi, s_{3}=r_{3} \pi$ be the first three simplex widths of $\mathbb{T}^{2} \times U$. Then $r_{1}, r_{2}, r_{3}$ must satisfy $0<r_{1} \leq 1$, $\frac{1}{2} r_{1} \leq r_{2} \leq r_{1}$, and

$\frac{1}{2} r_{1} \leq r_{3} \leq \min \left\{r_{2}, \max \left\{2-\left(r_{1}+r_{2}\right), \frac{1}{3}\left(r_{1}+r_{2}\right)\right\}\right\}$.

Conversely, if $r_{1}, r_{2}, r_{3}$ are chosen arbitrarily so that they satisfy the above inequalities, then there exists a $U$ and a volume-preserving diffeomorphism between $\mathbb{T}^{2} \times U$ and $\mathbb{T}^{2} \times \square(1)$ so that $s_{1}=r_{1} \pi, s_{2}=r_{2} \pi$, $s_{3}=r_{3} \pi$ are the first three simplex widths of $\mathbb{T}^{2} \times U$.

\section{PACKING CAPACITIES}

Let $\mathcal{M}^{2 n}$ denote the set of all $2 n$-dimensional symplectic manifolds. Throughout this paper, the focus will be on open subsets of $\left(\mathbb{R}^{2 n}, \omega_{0}\right)$, where $\omega_{0}=$ $d x_{1} \wedge d y_{1}+\cdots+d x_{n} \wedge d y_{n}$, and of the cotangent bundle of a torus $\left(T^{*} \mathbb{T}^{n}, \omega_{0}\right)$,

$T^{*} \mathbb{T}^{n}=\mathbb{T}^{n} \times \mathbb{R}^{n}=\frac{\left\{\left(x_{1}, \ldots, x_{n}\right)\right\}}{\pi \mathbb{Z}^{n}} \times\left\{\left(y_{1}, \ldots, y_{n}\right)\right\}$,

where $\omega_{0}=\sum d x_{i} \wedge d y_{i}$. Particular open sets of interest include $B^{2 n}, Z^{2 n} \subset\left(\mathbb{R}^{2 n}, \omega_{0}\right)$ and $P^{2 n}, Y^{2 n} \subset$ $\left(T^{*} \mathbb{T}^{n}, \omega_{0}\right)$ :

$$
\begin{aligned}
B^{2 n}(\sqrt{r}) & :=\left\{x_{1}^{2}+y_{1}^{2}+\cdots+x_{n}^{2}+y_{n}^{2}<r\right\}, \\
Z^{2 n}(\sqrt{r}) & :=\left\{\left(x_{1}, y_{1}, \ldots, x_{n}, y_{n}\right) \mid x_{1}^{2}+y_{1}^{2}<r\right\}, \\
P^{2 n}(r) & :=\mathbb{T}^{n} \times \triangle^{n}(r), \\
\triangle^{n}(r) & :=\left\{\left(y_{1}, \ldots, y_{n}\right) \mid 0<y_{i}, \sum y_{i}<r\right\}, \\
Y^{2 n}(r) & :=\mathbb{T}^{n} \times(0, r) \times\left(\mathbb{R}^{+}\right)^{n-1},
\end{aligned}
$$

where $\mathbb{R}^{+}:=(0, \infty)$. Recall the definition of $\mathcal{M}_{\mathbb{Z}^{n}} \subset$ $\mathcal{M}^{n}$ from (0.2). $P^{2 n}$ and $Y^{2 n}$ are the $\mathcal{M}_{\mathbb{Z}^{n}}$ "counterparts" of $B^{2 n}$ and $Z^{2 n}$ since, as shown in [Traynor 1995], there exist for any $\varepsilon>0$ symplectic embeddings

$$
\begin{aligned}
& B(\sqrt{r-\varepsilon}) \rightarrow P(r) \rightarrow B(\sqrt{r}), \\
& Z(\sqrt{r-\varepsilon}) \rightarrow Y(r) \rightarrow Z(\sqrt{r}) .
\end{aligned}
$$

A basic problem in symplectic topology is to find invariants of a symplectic manifold. It is easy to verify that the volume $\int_{M} \omega^{n}$ of the symplectic manifold $(M, \omega)$ is a symplectic invariant. In their search for periodic solutions of hamiltonian vector fields on convex energy surfaces in $\mathbb{R}^{2 n}$, Ekeland and Hofer [1989] defined a nontrivial invariant that they called a symplectic capacity. The concept of a symplectic capacity was generalized and axiomatized as follows.

Definition 2.2 (compare [Hofer and Zehnder 1994; McDuff and Salamon 1995]). Let $\mathcal{M}^{2 n}$ denote the set of $2 n$-dimensional symplectic manifolds. An $\mathcal{M}$ capacity is a function $c: \bigcup_{n \in \mathbb{N}} \mathcal{M}^{2 n} \rightarrow[0, \infty]$ satisfying:

Monotonicity: If there is a symplectic embedding

$$
\left(M_{1}, \omega_{1}\right) \rightarrow\left(M_{2}, \omega_{2}\right)
$$

where $M_{1}, M_{2} \in \mathcal{M}^{2 n}$, then $c\left(M_{1}, \omega_{1}\right) \leq c\left(M_{2}, \omega_{2}\right)$. Conformality: $c(M, \alpha \omega)=|\alpha| c(M, \omega)$, for all nonzero $\alpha \in \mathbb{R}$.

Nontriviality: $0<c\left(B^{2 n}(1), \omega_{0}\right)$ and $c\left(Z^{2 n}(1), \omega_{0}\right)$ is finite.

The nontriviality condition guarantees that, in dimensions greater than two, volume is not a capacity. The search for symplectic capacities and techniques to calculate them are major areas of research in symplectic topology.

For each $k$, the ball $k$-packing width $g_{k}(M)$ defined in (0.1) is a symplectic capacity. This can be proved using a slight variation of the proof that $g_{1}$ is a capacity as given in [Hofer and Zehnder 1994; McDuff and Salamon 1995]. It is not difficult to verify the monotonicity and conformality axioms. However, the nontriviality is difficult and is essentially equivalent to Gromov's nonsqueezing theorem.

Nonsqueezing Theorem 2.3 [Gromov 1985]. There is a symplectic embedding $\psi: B^{2 n}(s) \rightarrow Z^{2 n}(t)$ if and only if $s \leq t$.

Recall the definition of the simplex $k$-packing widths from (0.3). It is easy to check that $s_{k}$ is a symplectic invariant. It will be shown that the simplex $k$-widths satisfy $\mathcal{M}_{\mathbb{Z}^{n}}$ analogues of the capacity axioms.

Definition 2.4. An $\mathcal{M}_{\mathbb{Z}}$-capacity is a function $z$ from $\bigcup_{n} \mathcal{M}_{\mathbb{Z}^{n}}$ to $[0, \infty]$ satisfying:

Monotonicity: If there exists a symplectic embedding $\psi:\left(M_{1}, \omega_{1}\right) \rightarrow\left(M_{2}, \omega_{2}\right)$, where $M_{1}, M_{2} \in$ $\mathcal{M}_{\mathbb{Z}^{n}}$, such that $\psi_{*}: H_{1}\left(M_{1}\right) \stackrel{\simeq}{\longrightarrow} H_{1}\left(M_{2}\right)$, then $z\left(M_{1}, \omega_{1}\right) \leq z\left(M_{2}, \omega_{2}\right)$.

Conformality: $z(M, \alpha \omega)=|\alpha| z(M, \omega)$ for all nonzero $\alpha \in \mathbb{R}$.

Nontriviality: $0<z\left(P^{2 n}(1), \omega_{0}\right)$ and $z\left(Y^{2 n}(1), \omega_{0}\right)$ is finite. 
It is easy to verify that the $s_{k}$ satisfy the monotonicity and conformality axioms. The nontriviality can be deduced from Gromov's nonsqueezing theorem (see Remark 2.9) or, alternatively, as an easy consequence of the following theorem which will be proved at the end of this section.

Theorem 2.5. Let $U$ and $V$ be open, connected subsets of $\mathbb{R}^{n}$ with $H_{1}(U ; \mathbb{Z})=H_{1}(V ; \mathbb{Z})=0$. Consider $\mathbb{T}^{n} \times U, \mathbb{T}^{n} \times V \subset\left(T^{*} \mathbb{T}^{n}, \omega_{0}\right)$. Given embeddings

$$
\psi_{i}: \mathbb{T}^{n} \times U \rightarrow \mathbb{T}^{n} \times V, \quad \text { for } i=1, \ldots, k,
$$

satisfying the conditions $\psi_{i}^{*} \omega_{0}=\omega_{0}$,

$$
\left(\psi_{i}\right)_{*}: H_{1}\left(\mathbb{T}^{n} \times U\right) \stackrel{\simeq}{\longrightarrow} H_{1}\left(\mathbb{T}^{n} \times V\right),
$$

and whose images are pairwise disjoint, there exist $M_{i} \in \operatorname{GL}(n, \mathbb{Z})$ and $t_{i} \in \mathbb{R}^{n}$, for $i=1, \ldots, k$, satisfying $M_{i} U+t_{i} \subset V$ and such that the images $M_{i} U+t_{i}$ are pairwise disjoint.

As a corollary, for some subsets of $\left(T^{*} \mathbb{T}^{n}, \omega_{0}\right)$, the invariant $s_{k}$ has the following "algorithmic definition".

Corollary 2.6. Suppose $V$ is an open, connected subset of $\mathbb{R}^{n}$ with $H_{1}(V ; \mathbb{Z})=0$. Then

$s_{k}\left(\mathbb{T}^{n} \times V, \omega_{0}\right)=\sup \{\pi r \mid$ there are $k$ integer-affine

transformations taking $\triangle(r)$ into $V$ and having pairwise disjoint images $\}$.

Remark 2.7. It is easy to show that every $k$-tuple of integer-affine transformations with the stated properties gives a lower bound for $s_{k}\left(\mathbb{T}^{n} \times V, \omega_{0}\right)$. Given $M \in \mathrm{GL}(n, \mathbb{Z}), t \in \mathbb{R}^{n}$,

$$
\psi_{(M, t)}(x, y):=\left(\left(M^{-1}\right)^{T} x, M y+t\right)
$$

is a symplectic embedding of $P^{2 n}(r)$ into $T^{*} \mathbb{T}^{n}$. If $M\left(\triangle^{n}(r)\right)+t \subset V$ then $\psi_{(M, t)}\left(P^{2 n}(r)\right) \subset \mathbb{T}^{n} \times V$ and $s_{1}\left(\mathbb{T}^{n} \times V\right) \geq \pi r$. Similarly, finding $M_{1}, \ldots, M_{k} \in$ $\operatorname{GL}(n, \mathbb{Z})$ and $t_{1}, \ldots, t_{k} \in \mathbb{R}^{n}$ such that all the images $M_{i}\left(\triangle^{n}(r)\right)+t_{i}$ are contained in $V$ and pairwise disjoint implies $s_{k}\left(\mathbb{T}^{n} \times V\right) \geq \pi r$. If $k \operatorname{Vol} \triangle^{n}(r)=$ Vol $U$, it is possible to conclude also that $s_{k}\left(\mathbb{T}^{n} \times V\right)$ equals $\pi r$.

An " $\mathcal{N}_{\mathbb{Z}}$-nonsqueezing theorem" is another easy consequence of Theorem 2.5.

Corollary 2.8 $\left(\mathcal{M}_{\mathbb{Z}}\right.$-Nonsqueezing). There is a symplectic embedding $\psi: P^{2 n}(s) \rightarrow Y^{2 n}(r)$ with

$$
\psi_{*}: H_{1}(P(s)) \stackrel{\simeq}{\longrightarrow} H_{1}(Y(r))
$$

if and only if $s \leq r$.

Proof. First, if $s \leq r$, the inclusion is a symplectic embedding $P^{2 n}(s) \rightarrow Y^{2 n}(r)$. Conversely, suppose there exists a symplectic embedding $\varphi: P^{2 n}(s) \rightarrow$ $Y^{2 n}(r)$. By Theorem 2.5, there exists $M \in \operatorname{GL}(n, \mathbb{Z})$ and $t \in \mathbb{R}^{n}$ so that $M(\triangle)+t \subset(0, r) \times\left(\mathbb{R}^{+}\right)^{n-1}$. Let $\rho_{1}: \mathbb{R}^{n} \rightarrow \mathbb{R}$ denote the projection to the first coordinate. Then

$$
\begin{aligned}
& \left|\rho_{1}(M(\triangle(s))+t)\right| \\
& \quad:=\sup \left\{\left|\rho_{1}(z)-\rho_{1}(w)\right|: z, w \in M(\triangle(s))+t\right\}
\end{aligned}
$$

is a positive integral multiple of $s$. Since

$$
\rho_{1}(M(\triangle(s))+t) \subset(0, r),
$$

we have $\left|\rho_{1}(M(\triangle(s)+t))\right| \leq r$ and thus $s \leq r$.

Remark 2.9. This theorem can alternatively be proved using Gromov's Nonsqueezing Theorem 2.3. For suppose there exists an embedding $\psi: P^{2 n}(s) \rightarrow$ $Y^{2 n}(r)$ when $s>r$. Choose $\varepsilon<s-r$. By (2.1), there exist embeddings $B^{2 n}(\sqrt{s-\varepsilon}) \rightarrow P^{2 n}(s) \rightarrow$ $Y^{2 n}(r) \rightarrow Z^{2 n}(\sqrt{r})$, a contradiction to Theorem 2.3 .

Corollary 2.10. $s_{k}(M)$ is an $\mathcal{M}_{\mathbb{Z}}$-capacity.

The remainder of the section is devoted to proving Theorem 2.5. The rigidity of the packings is related to the rigidity of exact lagrangian submanifolds. A lagrangian submanifold of $\left(M^{2 n}, \omega\right)$ is an $n$-dimensional manifold $L$ such that $\omega(v, w)=0$, $\forall v, w \in T_{p} L, \forall p \in L$. If $\omega=-d \lambda$, this implies $i^{*}(\lambda) \in H^{1}(L)$ is a closed 1-form, where $i: L \rightarrow M$ is the inclusion map. Fixing $\lambda, L$ is called an exact lagrangian if $i^{*}(\lambda)$ is an exact form: $i^{*} \lambda=d f$, with $f: L \rightarrow \mathbb{R}$. A deep and important fact in symplectic topology is that exact lagrangians must intersect.

Theorem 2.11 (Intersections of exact tori) [Gromov 1985; Lalonde and Sikorav 1991]. If $L, L^{\prime}$ are closed, exact lagrangian tori in $T^{*} \mathbb{T}^{n}$ then $L \cap L^{\prime} \neq \varnothing$.

Remark. This intersection theorem was proved using the technique of holomorphic curves by Gromov for more general cotangent bundles in the cases where $L^{\prime}$ is the zero section or $L^{\prime}$ is hamiltonian isotopic to $L$. A deep study which further extended the cases where lagrangian intersections must occur was done in [Lalonde and Sikorav 1991]. The proof of Theorem 2.5 needs only the case of lagrangian tori. 
The following notion of strong exactness is an important ingredient in proving the rigidity statements.

Definition 2.12 [Sikorav 1989]. Let $\mathcal{U}$ be an open subset of $T^{*} \mathbb{T}^{n}=\mathbb{T}^{n} \times \mathbb{R}^{n}$ with its standard symplectic form $\omega_{0}=-d \lambda$, where $\lambda=\sum_{i} y_{i} d x_{i}$. A symplectic embedding of $\mathcal{U}$ into $\mathbb{T}^{n} \times \mathbb{R}^{n}$ is strongly exact if

(a) $\left[\psi^{*} \lambda-\lambda\right]=0 \in H^{1}(\mathcal{U} ; \mathbb{R})$, and

(b) $\psi^{*}=i^{*}: H^{1}\left(\mathbb{T}^{n} \times \mathbb{R}^{n} ; \mathbb{R}\right) \rightarrow H^{1}(\mathcal{U} ; \mathbb{R})$, where $i$ is the inclusion.

Remark. A hamiltonian diffeomorphism is always a strongly exact embedding.

If $L$ is an exact lagrangian submanifold then, for an arbitrary symplectic diffeomorphism $\psi, \psi(L)$ is a lagrangian submanifold but not necessarily exact. For example, $\mathbb{T}^{n} \times\{u\}$ is an exact lagrangian submanifold of $T^{*} \mathbb{T}^{n}$ only when $u=0$. However, it is easy to check that exact lagrangians are preserved under strongly exact symplectic embeddings. In addition, strongly exact embeddings are preserved under conjugation. In particular, let $\tau_{u}$ be a translation by $u$ in the fibers of $T^{*} \mathbb{T}^{n}: \tau_{u}(x, y)=(x, y+u)$. Then if $\psi: \mathbb{T}^{n} \times U \rightarrow \mathbb{T}^{n} \times \mathbb{R}^{n}$ is a strongly exact embedding, for each $u \in U$,

$$
\tau_{u}^{-1} \circ \psi\left(\mathbb{T}^{n} \times\{u\}\right)=\tau_{u}^{-1} \circ \psi \circ \tau_{u}\left(\mathbb{T}^{n} \times\{0\}\right)
$$

is an exact lagrangian submanifold.

The next theorem demonstrates the rigidity of strongly exact embeddings.

Theorem 2.13 [Sikorav 1989]. Let $U, V$ be open subsets of $\mathbb{R}^{n}$ and consider $\mathbb{T}^{n} \times U, \mathbb{T}^{n} \times V \subset\left(T^{*} \mathbb{T}^{n}, \omega_{0}\right)$. If there exists a strongly exact embedding of $\mathbb{T}^{n} \times U$ into $\mathbb{T}^{n} \times V$ then $U \subset V$.

Using this, Sikorav easily proved the surprising result that if $U, V \subset \mathbb{R}^{n}$ are connected, open subsets with $H^{1}(U)=H^{1}(V)=0$ (either real or integer coefficients) then $\mathbb{T}^{n} \times U$ is symplectically equivalent to $\mathbb{T}^{n} \times V$ if and only if there exists $M \in \operatorname{GL}(n, \mathbb{Z})$ and $t \in \mathbb{R}^{n}$ such that $M(U)+t=V$. Sikorav's proof of this fact can be modified to prove Theorem 2.5.

Proof of Theorem 2.5. Suppose that $\left\{\varphi_{i}\right\}_{i=1}^{k}$ satisfy the hypotheses of the theorem. The conditions on $U, V$ imply $H_{1}\left(\mathbb{T}^{n} \times W\right)=H_{1}\left(\mathbb{T}^{n}\right)$ for $W=U, V$ and either real or integral coefficients. By the universal coefficient theorem,

$$
H^{1}\left(\mathbb{T}^{n} ; \mathbb{Z}\right)=\operatorname{Hom}\left(H_{1}\left(\mathbb{T}^{n}\right) ; \mathbb{Z}\right) .
$$

Thus the hypotheses on $\varphi_{i}$ imply that the

$$
\left(\varphi_{i}\right)^{*}: H^{1}\left(\mathbb{T}^{n} \times V ; \mathbb{Z}\right) \rightarrow H^{1}\left(\mathbb{T}^{n} \times U ; \mathbb{Z}\right)
$$

are isomorphisms. Associate $M_{i}\left(\varphi_{i}\right) \in \operatorname{GL}(n, \mathbb{Z})$ and $t_{i}\left(\varphi_{i}\right) \in \mathbb{R}^{n}$ as follows.

(a) Choose a fixed identification of $\operatorname{Aut}\left(H^{1}\left(\mathbb{T}^{n} ; \mathbb{Z}\right)\right)$ with $\operatorname{GL}(n, \mathbb{Z})$. Then, by hypothesis,

$$
M_{i}\left(\varphi_{i}\right):=\varphi_{i}^{*} \in \operatorname{Aut}\left(H^{1}\left(\mathbb{T}^{n} ; \mathbb{Z}\right)\right)=\operatorname{GL}(n, \mathbb{Z})
$$

for $i=1, \ldots, k$.

(b) Since $\varphi_{i}$ is symplectic,

$$
t_{i}\left(\varphi_{i}\right):=\left[\varphi_{i}^{*} \lambda-\lambda\right] \in H^{1}\left(\mathbb{T}^{n} \times U ; \mathbb{R}\right) \simeq \mathbb{R}^{n} .
$$

A direct calculation shows that

$\psi_{i}:=\varphi_{i} \circ \varphi_{M_{i}, t_{i}}^{-1}: \mathbb{T}^{n} \times\left(M_{i} U+t_{i}\right) \rightarrow \operatorname{Im} \varphi_{i} \subset \mathbb{T}^{n} \times V$ is strongly exact, where $\varphi_{M_{i}, t_{i}}$ is defined as in Remark 2.7. The proof will be finished by proving that the domains of these $\psi_{i}$ give a packing of $\mathbb{T}^{n} \times V$. Since for all $i, \psi_{i}$ is strongly exact, Theorem 2.13 implies $M_{i} U+t_{i} \subset V$. Next suppose there exists $c \in\left(M_{i} U+t_{i}\right) \cap\left(M_{j} U+t_{j}\right)$ where $i \neq j$. Consider $\tau_{c}: \mathbb{T}^{n} \times \mathbb{R}^{n} \rightarrow \mathbb{T}^{n} \times \mathbb{R}^{n}$ defined by

$$
\tau_{c}(x, y)=(x, y+c) \text {. }
$$

Then

$$
\begin{aligned}
& T_{i}:=\tau_{c}^{-1} \circ \psi_{i} \circ \tau_{c}\left(\mathbb{T}^{n} \times\{0\}\right), \\
& T_{j}:=\tau_{c}^{-1} \circ \psi_{j} \circ \tau_{c}\left(\mathbb{T}^{n} \times\{0\}\right)
\end{aligned}
$$

are exact lagrangian tori in $T^{n} \times \mathbb{R}^{n}$, so $T_{i} \cap T_{j} \neq \varnothing$ by Theorem 2.11. This implies $\tau_{c}\left(T_{i}\right) \cap \tau_{c}\left(T_{j}\right) \neq \varnothing$. However, since $\tau_{c}\left(T_{i}\right) \subset \operatorname{Im} \varphi_{i}$ and $\tau_{c}\left(T_{j}\right) \subset \operatorname{Im} \varphi_{j}$, this is a contradiction.

Remark 2.14. In fact, a version of Theorem 2.5 holds for $n=2$ under weaker conditions on the first homology of the target space. Namely, consider the modification of 2.5 where $V=D^{2} \backslash\{0\}$, where $D^{2}$ is the open disc centered at the origin, and

$$
\left(\psi_{i}\right)_{*}: H_{1}\left(\mathbb{T}^{2} \times U\right) \rightarrow H_{1}\left(\mathbb{T}^{2} \times V\right)
$$

is injective, for $i=1, \ldots, k$. Then each lagrangian torus $\psi_{i}\left(\mathbb{T}^{n} \times\{p\}\right)$ is incompressible in $\mathbb{T}^{2} \times V$ and thus, by a result in [Giroux 1994], must be homotopic to a "horizontal" torus of the form $\mathbb{T}^{2} \times\{\star\}$, with $\star \in V$. Thus $\psi_{i}^{*}$ can again be identified with an element of $\operatorname{Aut}\left(H_{1}\left(\mathbb{T}^{n}\right)\right)=\operatorname{GL}(n, \mathbb{Z})$, and the proof of Theorem 2.5 follows through. It would be interesting to understand more examples where analogues of Theorem 1.1 hold. 


\section{PACKING ANALOGUES OF BALLS AND ELLIPSOIDS}

As described in Section $1, P^{n}(r)=\mathbb{T}^{n} \times \triangle^{n}(r) \subset$ $T^{*} \mathbb{T}^{n}$ can be thought of as an analogue of $B^{2 n}(\sqrt{r})$. The idea for the following packing constructions is to start with a configuration as found in a packing with $k^{2}$ triangles. This full packing is then slit along a row and shrunk until it is possible to insert "skinny" triangles. In the terminology of Section 5, these are packings with two convex lattice polytopes. See Figure 1.7. By an easy construction, the lower bound given by Theorem 3.1 for packing 6 simplices is not optimal. See Figure 1.4(c). By the computer results in Theorems 1.2 and 1.8, the lower bounds in this theorem are known to be optimal for packing 5, 10, and 11 simplices, and are conjectured optimal for packing 17 and 18 simplices.

Theorem 3.1. For $k \geq 2$,

$$
s_{k^{2}+1}\left(P^{4}(1)\right) \geq \frac{k}{k^{2}+1} \pi
$$

and

$$
s_{k^{2}+2}\left(P^{4}(1)\right) \geq \frac{k-1}{k^{2}-k+1} \pi .
$$

Proof. Let $r=k /\left(k^{2}+1\right)$. The following elements $M_{i} \in \mathrm{GL}(2, \mathbb{Z})$ and $t_{i} \in \mathbb{R}^{2}$ give a $k^{2}+1$ packing of $\triangle(1)$ by $\triangle(r)$ and thus, by Remark 2.7 , a simplex packing of $P^{4}(1)$. The images of $\triangle(r)$ lie in $k+1$ "rows". The bottom row contains the following $k+1$ images of $\triangle(r)$ :

$$
\begin{array}{ll}
M_{1}^{1}=\left(\begin{array}{ll}
1 & 0 \\
0 & 1
\end{array}\right), & t_{1}^{1}=\left(\begin{array}{l}
0 \\
0
\end{array}\right) ; \\
M_{2}^{1}=\left(\begin{array}{rr}
-1 & 0 \\
0 & -1
\end{array}\right), & t_{2}^{1}=\left(\begin{array}{l}
r \\
r
\end{array}\right) ; \\
M_{i}^{1}=\left(\begin{array}{cc}
i-3 & i-2 \\
-1 & -1
\end{array}\right), & t_{i}^{1}=\left(\begin{array}{l}
r \\
r
\end{array}\right)
\end{array}
$$

for $i=3, \ldots, k+1$. The next row contains $k-1$ images of $\triangle(r)$ :

$$
M_{i}^{2}=\left(\begin{array}{cc}
1 & k-i+1 \\
0 & -1
\end{array}\right), \quad t_{i}^{2}=\left(\begin{array}{c}
i-1 \\
r+r / k
\end{array}\right)
$$

for $i=1, \ldots, k-1$. These $2 k$ triangles are all contained in

$$
\triangle(1) \cap\left\{\left(y_{1}, y_{2}\right) \mid y_{2}<r+\frac{r}{k}=\frac{k+1}{k^{2}+1}\right\} .
$$

Since $\triangle(1) \cap\left\{y_{2}>(k+1) /\left(k^{2}+1\right)\right\}$ is a right, isosceles triangle with legs of length $(k-1) r$, it can be fully packed using $(k-1)^{2}$ copies of $\triangle(r)$. Altogether, there are $(k+1)+(k-1)+(k-1)^{2}=k^{2}+1$ disjoint images of $\triangle(r)$.

To estimate $s_{k^{2}+2}\left(P^{4}(1)\right)$, consider

$$
r=\frac{k-1}{k^{2}-k+1},
$$

and let $M_{i}^{j} \in \mathrm{GL}(2, \mathbb{Z})$ and $t_{i}^{j} \in \mathbb{R}^{2}$ be as follows. Again, the images of $\triangle(r)$ lie in one of $k+1$ "rows". The bottom row of $\triangle(1)$ has the same $k+1$ embeddings as the $k^{2}+1$ packing. In the row above the bottom row, insert $k$ triangles:

$$
M_{i}^{2}=\left(\begin{array}{cc}
1 & k-i+1 \\
0 & -1
\end{array}\right), \quad t_{i}^{2}=\left(\begin{array}{c}
i-1 \\
r+r / k
\end{array}\right)
$$

for $i=1, \ldots, k-1$, and

$$
M_{k}^{2}=\left(\begin{array}{cc}
k & k-1 \\
-1 & -1
\end{array}\right), \quad t_{k}^{2}=\left(\begin{array}{c}
0 \\
r+r /(k-1)
\end{array}\right) .
$$

Again, these $2 k+1$ triangles are all contained in

$$
\triangle(1) \cap\left\{y_{2}<r+\frac{r}{k}=\frac{k+1}{k^{2}+1}\right\} .
$$

Since $\triangle(1) \cap\left\{y_{2}>r+r / k\right\}$ is a right, isosceles triangle with legs of length $(k-1) r$, it can be fully packed with an additional $(k-1)^{2}$ triangles. Altogether, this makes a packing with $(k+1)+(k)+(k-1)^{2}=$ $k^{2}+2$ copies of $\triangle(r)$.

Remark. A similar idea was employed in [Krouglikov $1994]$ to construct ball packings of $B^{4}(1)$ before Biran proved there exist full packings. Krouglikov proved that

$$
g_{k^{2}+1}\left(B^{4}(1)\right) \geq \frac{3 k}{3 k^{2}+k}
$$

when $k \geq 3$. These estimates on $g_{k}$ are weaker than those given as a corollary to Theorem 3.1.

In arbitrary dimensions, Gromov [1985] proved that $g_{k}\left(B^{2 n}(1)\right) \leq \frac{1}{2} \pi$, for $1<k \leq 2^{n}$. The following theorem is implied by this result. The proof given uses euclidean rather than algebraic geometry.

Theorem 3.2. $s_{k}\left(P^{2 n}(1)\right) \leq \frac{1}{2} \pi$ for $1<k \leq 2^{n}$.

Remark. By conformality (Definition 2.4), this statement is equivalent to $s_{k}\left(P^{2 n}(\alpha)\right) \leq \frac{1}{2} \alpha \pi$ when $1<$ $k \leq 2^{n}$. The estimate that $s_{k}\left(P^{2 n}(1)\right) \leq \frac{1}{2} \pi$ holds for all $k>1$, but when $k>2^{n}$ the volume constraint is more restrictive. If for any $j>1$, it is known that $s_{j}\left(P^{2 n}(1)\right)=\frac{1}{2} \pi$ then it follows that 
$s_{k}\left(P^{2 n}(1)\right)=\frac{1}{2} \pi$ for $1<k \leq j$. An interesting and natural attempt at a full ball packing construction for $B^{2 n}(1)$ in [Krouglikov 1994] would have led to a full simplex $2^{n}$-packing of $P^{2 n}(1)$. However, the inductive argument is incomplete. It is not hard to check that $s_{n+1}\left(P^{2 n}(1)\right)=\frac{1}{2} \pi$. This can be seen by constructing embeddings that have images in the $n+1$ "corners". More precisely, let $M_{i}=\mathbb{I}^{n}$ for $1, \ldots, n+1, t_{n+1}=\mathbf{0}$, and $t_{i}=\left(0, \ldots, 0, \frac{1}{2}, 0, \ldots, 0\right)$ for $i=1, \ldots n$. Thus

$$
s_{k}\left(P^{2 n}(1)\right)=\frac{1}{2} \pi \quad \text { for } 1<k \leq n+1 .
$$

In [Mastrangeli 1997], it is shown that for $n=3,4$, there is a full packing of $P^{2 n}(1)$ with $2^{n}$ copies of $P^{2 n}\left(\frac{1}{2}\right)$, and thus

$s_{k}\left(P^{2 n}(1)\right)=\frac{1}{2} \pi \quad$ for $1<k \leq 2^{n}$ when $n=3,4$.

It is currently work in progress by Mastrangeli to generalize this to all $n$.

Before proving Theorem 3.2, some tools will be built which are based on the restrictive nature of the image of $\triangle^{n}(r)$ under $\operatorname{GL}(n, \mathbb{Z})$. Given any symplectic embedding $\psi: P^{4}(r) \rightarrow T^{*} \mathbb{T}^{n}$ such that $\psi_{*}: H_{1}\left(P^{2 n}(r)\right) \stackrel{\simeq}{\longrightarrow} H_{1}\left(T^{*} \mathbb{T}^{n}\right)$, we can identify $\psi_{*}$ with an element of $\mathrm{GL}(n, \mathbb{Z})$. This automorphism is reflected in the shape of the triangle. An equivalence relation on $\operatorname{GL}(n, \mathbb{Z})$ will be given by the shape (up to translation) of the image $M\left(\triangle^{n}(r)\right.$ ); $M \in \mathrm{GL}(n, \mathbb{Z})$.

Definition 3.3. Let $M_{1}, M_{2} \in \mathrm{GL}(n, \mathbb{Z})$. Then $M_{1} \equiv$ $M_{2}$ if and only if there exists $t \in \mathbb{R}^{n}$ such that $M_{1}\left(\triangle^{n}(r)\right)+t=M_{2}\left(\triangle^{n}(r)\right)$. Equivalence classes will be denoted by $[M]$.

The following lemma emphasizes the important role played by $r=\frac{1}{2}$.

Lemma 3.4. If $r>\frac{1}{2}$ and $\psi: P^{2 n}(r) \rightarrow P^{2 n}(1)$ is a symplectic embedding with

$$
\psi_{*}: H_{1}\left(P^{2 n}(r)\right) \stackrel{\simeq}{\longrightarrow} H_{1}\left(P^{2 n}(1)\right)
$$

then $\left[\psi_{*}\right]=\left[\mathbb{I}^{n}\right]$.

Proof. Since $M \in \mathrm{GL}(n, \mathbb{Z})$, the length

$$
\left|\rho_{i}\left(M\left(\triangle^{n}(r)\right)+t\right)\right|
$$

of the projection of $M\left(\triangle^{n}(r)\right)+t$ onto the $y_{i}$ axis is a positive integral multiple of $r$. The images of these triangles lie in $\triangle^{n}(1)$, for each $i$, and hence
$\left|\rho_{i}\left(M\left(\triangle^{n}(r)\right)+t\right)\right|=m r \leq 1$, for some $m \in \mathbb{N}$. However, $r>\frac{1}{2}$ then implies that $m=1$. Hence the vertices of $M\left(\triangle^{n}(r)\right)+t$ must coincide with $n+1$ vertices of a translated $n$-dimensional cube of size $r$. A vertex $\left(y_{1}, \ldots, y_{n}\right)$ of $M\left(\triangle^{n}(r)\right)+t \subset \triangle^{n}(1)$ must satisfy $y_{i} \geq 0$ and $\sum_{i=1}^{n} y_{i} \leq 1$. Thus if $r>\frac{1}{2}$, there are at most $n+1$ vertices of the translated cube that lie in $\triangle^{n}(1)$. Hence $M\left(\triangle^{n}(r)\right)+t$ is a subset of $\triangle^{n}(1)$ only if it is the interior of the convex hull of $\left\{\left(t_{1}, \ldots, t_{n}\right),\left(t_{1}+r, t_{2}, \ldots, t_{n}\right),\left(t_{1}, t_{2}+r, \ldots, t_{n}\right)\right.$, $\left.\ldots,\left(t_{1}, t_{2}, \ldots, t_{n}+r\right)\right\}$, for $\sum t_{i} \leq 1-r$. Therefore $\left[\psi_{*}\right]=\left[\mathbb{I}^{n}\right]$.

The image of $\triangle^{n}(r)$ under an element of $\operatorname{GL}(n, \mathbb{Z})$ will always be convex. To study more than one embedding, it is convenient to look at the translation set associated with each shape.

Definition 3.5. Let $T$ and $R$ be open sets in $\mathbb{R}^{n}$. The translation set of $T$ in $R$ is $\tau(T, R):=\left\{t \in \mathbb{R}^{n} \mid\right.$ $T+t \subset R\}$.

Example 3.6. It is easy to check that $\tau\left(\triangle^{n}\left(\frac{1}{2}\right), \triangle^{n}(1)\right)$ is the closed simplex with the $n+1$ vertices $(0, \ldots, 0)$, $\left(\frac{1}{2}, 0, \ldots, 0\right), \ldots,\left(0, \ldots, 0, \frac{1}{2}\right)$.

The set $\tau(T, R)$ will always be closed. For the cases of interest, it will also always be convex.

Lemma 3.7. If $T=M(\triangle(r)), M \in \mathrm{GL}(n, \mathbb{Z})$, and $R$ is a convex subset of $\mathbb{R}^{n}$ then $\tau(T, R)$ is convex.

Proof. Let $t_{1}, t_{2} \in \tau$ and $\alpha_{1}, \alpha_{2} \geq 0$ such that $\alpha_{1}+$ $\alpha_{2}=1$. Then $T+t_{1}, T+t_{2} \subset R$ and $R$ convex implies that $T+\alpha_{1} t_{1}+\alpha_{2} t_{2}=\left(\alpha_{1}+\alpha_{2}\right) T+\alpha_{1} t_{1}+\alpha_{2} t_{2}=$ $\left(\alpha_{1}\left(T+t_{1}\right)+\alpha_{2}\left(T+t_{2}\right)\right) \subset R$. Hence $\tau(T, R)$ is convex.

The following lemma will be used to prove Propositions 3.9 and 4.3 .

Lemma 3.8. Let $T=M(\triangle(r))$ and $M \in \mathrm{GL}(n, \mathbb{Z})$. If $p_{i} \in T+z_{i}$ for $i=1,2, \ldots, l$, then for any $s_{1}, \ldots, s_{l}$ that satisfy $s_{i} \geq 0$ and $\sum_{i=1}^{l} s_{i}=1$, we have

$$
\sum_{i=1}^{l} s_{i} p_{i} \in T+\sum_{i=1}^{l} s_{i} z_{i}
$$

Proof. Let $m=n+1$ and suppose

$$
T=\left\{\sum_{j=1}^{m} t_{j} y_{j} \mid t_{j}>0, \sum_{j=1}^{m} t_{j}=1\right\} .
$$


Since $p_{i} \in T+z_{i}$, there exist $t_{j}^{i}$ such that $p_{i}$ equals $\sum_{j=1}^{m} t_{j}^{i} y_{j}+z_{i}$, where $t_{j}^{i}>0$ and $\sum_{j=1}^{m} t_{j}^{i}=1$. Then

$$
\begin{aligned}
\sum_{i=1}^{l} s_{i} p_{i} & =\sum_{i=1}^{l}\left(\sum_{j=1}^{m} s_{i} t_{j}^{i} y_{j}+s_{i} z_{i}\right) \\
& =\sum_{i=1}^{l} \sum_{j=1}^{m} s_{i} t_{j}^{i} y_{j}+\sum_{i=1}^{l} s_{i} z_{i} .
\end{aligned}
$$

To verify that this is an element of $T+\sum_{i=1}^{l} s_{i} z_{i}$, first note that

$$
\sum_{i=1}^{l} \sum_{j=1}^{m} s_{i} t_{j}^{i}=\sum_{i=1}^{l} s_{i} \sum_{j=1}^{m} t_{j}^{i}=\sum_{i=1}^{l} s_{i}=1 .
$$

Moreover the coefficients $\sum_{i=1}^{l} s_{i} t_{j}^{i}$ of each $y_{j}$ are strictly greater than zero since at least one $s_{i}$ is strictly greater than zero and $t_{j}^{i}$ are positive.

The next proposition will be useful to prove that there does not exist a packing containing two triangles of the same shape. It reduces checking all possible translations to the "extreme" vertex translations.

Proposition 3.9 (Same-shape noncompatibility). Let $T=$ $M(\triangle(r)), M \in \mathrm{GL}(n, \mathbb{Z})$, and let $\tau$ be the convex hull of $z_{1}, \ldots, z_{l}$ :

$$
\tau=\left\{\sum s_{i} z_{i} \mid s_{i} \geq 0, \quad \sum s_{i}=1\right\} .
$$

If the vertex translations satisfy $\left(T+z_{i}\right) \cap\left(T+z_{j}\right) \neq$ $\varnothing, \forall i, j=1, \ldots, l$, then for all $\alpha, \beta \in \tau,(T+\alpha) \cap$ $(T+\beta) \neq \varnothing$.

Proof. Let $\alpha, \beta \in \tau$. Then $\alpha=\sum_{i=1}^{l} r_{i} z_{i}, \beta=$ $\sum_{j=1}^{l} s_{j} z_{j}$ where $r_{i}, s_{j} \geq 0, \sum_{i=1}^{l} r_{i}=1=\sum_{j=1}^{l} s_{j}$. By hypothesis, there exists $p_{i j} \in\left(T+y_{i}\right) \cap\left(T+y_{j}\right)$ for $i, j=1, \ldots, l$. For $i=1, \ldots, l$, consider $q_{i}=$ $\sum_{j=1}^{l} s_{j} p_{i j}$ where $s_{j}$ are chosen from the definition of $\beta$. For fixed $i$, since $p_{i j} \in T+z_{i}$ for all $j$ and $T+z_{i}$ is convex, it follows that $q_{i} \in T+z_{i}$. By Lemma 3.8, $q_{i} \in T+\beta$. Thus $q_{i} \in\left(T+z_{i}\right) \cap(T+\beta)$. A similar argument shows that $\sum_{i=1}^{l} r_{i} q_{i} \in(T+\alpha) \cap(T+\beta)$, and thus $(T+\alpha) \cap(T+\beta) \neq \varnothing$.

Proof of Theorem 3.2. It suffices to prove that for $r>\frac{1}{2}$, if $\psi_{1}, \psi_{2}: P(r) \rightarrow P(1)$ are symplectic embeddings with $\left(\psi_{i}\right)_{*}: H_{1}(P(r)) \stackrel{\simeq}{\longrightarrow} H_{1}(P(1))$ then $\operatorname{Im} \psi_{1} \cap \operatorname{Im} \psi_{2} \neq \varnothing$. By Lemma 3.4, if $r>\frac{1}{2}$ then $\left[\psi_{*}\right]=\left[\mathbb{I}^{n}\right]$. From Example 3.6, the translation set $\tau\left(\triangle^{n}(r), \triangle^{n}(1)\right)$ has vertices $z_{0}=(0, \ldots, 0)$, $z_{1}=(1-r, 0, \ldots, 0), z_{2}=(0,1-r, 0, \ldots, 0), \ldots$, $z_{n}=(0,0, \ldots, 1-r)$. Then, for positive $\varepsilon$ less than $\frac{1}{2}(2 r-1) /(n-1) \leq \frac{1}{2}$, we have

$$
\left(\varepsilon, \ldots, \varepsilon, \frac{1}{2}, \varepsilon, \ldots, \varepsilon\right) \in\left(T+z_{0}\right) \cap\left(T+z_{i}\right)
$$

where $\frac{1}{2}$ is in the $i$-th position, and

$$
\begin{array}{r}
\left(\frac{\varepsilon}{n-2}, \ldots, \frac{\varepsilon}{n-2}, \frac{1}{2}-\varepsilon, \frac{\varepsilon}{n-2}, \ldots, \frac{1}{2}-\varepsilon, \frac{\varepsilon}{n-2}, \ldots, \frac{\varepsilon}{n-2}\right) \\
\in\left(T+z_{i}\right) \cap\left(T+z_{j}\right)
\end{array}
$$

where the $\frac{1}{2}-\varepsilon$ entries are the $i$-th and $j$-th. By Proposition 3.9, for all $\alpha, \beta \in \tau\left(\triangle^{n}(r), \triangle^{n}(1)\right)$, the intersection $\left(\triangle^{n}(r)+\alpha\right) \cap\left(\triangle^{n}(r)+\beta\right)$ is nonempty. Hence, if $r>\frac{1}{2}$, there do not exist two disjoint images of $\triangle^{n}(r)$ under $\mathrm{GL}(n, \mathbb{Z})$ and translations contained in $\triangle^{n}(1)$. Therefore, by Corollary 2.6, $s_{k}\left(P^{2 n}(1)\right) \leq \frac{1}{2} \pi$.

Next consider

$$
P\left(\alpha_{1}, \ldots, \alpha_{n}\right):=\mathbb{T}^{n} \times \triangle\left(\alpha_{1}, \ldots, \alpha_{n}\right),
$$

where $\triangle\left(\alpha_{1}, \ldots, \alpha_{n}\right)$ is the simplex with the $(n+1)$ vertices $(0, \ldots, 0),\left(\alpha_{1}, 0, \ldots, 0\right), \ldots,\left(0, \ldots, 0, \alpha_{n}\right)$. Recall, as described in Section 1, this is an analogue of an ellipsoid.

Theorem 3.10. Assume $\alpha_{1} \leq \alpha_{2} \leq \cdots \leq \alpha_{n}$. Then

$$
\begin{aligned}
& s_{1}\left(P\left(\alpha_{1}, \ldots, \alpha_{n}\right)\right)=\alpha_{1} \pi, \\
& s_{2}\left(P\left(\alpha_{1}, \ldots, \alpha_{n}\right)\right)=\min \left\{\alpha_{1}, \frac{1}{2} \alpha_{n}\right\} \pi .
\end{aligned}
$$

Proof. Notice that $P\left(\alpha_{1}, \ldots, \alpha_{n}\right) \subset Y^{2 n}\left(\alpha_{1}\right)$ and $P\left(\alpha_{1}, \ldots, \alpha_{n}\right) \subset P^{2 n}\left(\alpha_{n}\right)$. Thus, by monotonicity (Definition 2.4),

$s_{k}\left(P\left(\alpha_{1}, \ldots, \alpha_{n}\right)\right) \leq \min \left\{s_{k}\left(Y^{2 n}\left(\alpha_{1}\right)\right), s_{k}\left(P^{2 n}\left(\alpha_{n}\right)\right\}\right.$

for all $k$. In particular, Corollary 2.8 and Theorem 3.2 imply that $s_{1}\left(P\left(\alpha_{1}, \ldots, \alpha_{n}\right)\right) \leq \min \left\{\alpha_{1}, \alpha_{n}\right\} \pi=$ $\alpha_{1} \pi$ and $s_{2}\left(P\left(\alpha_{1}, \ldots, \alpha_{n}\right)\right) \leq \min \left\{\alpha_{1}, \frac{1}{2} \alpha_{n}\right\} \pi$. Since $P^{n}\left(\alpha_{1}\right) \subset P\left(\alpha_{1}, \ldots, \alpha_{n}\right), s_{1}\left(P\left(\alpha_{1}, \ldots, \alpha_{n}\right)\right) \geq \alpha_{1} \pi$ and thus the claim about $s_{1}$ follows. It will be shown that $s_{2}\left(P\left(\alpha_{1}, \ldots, \alpha_{n}\right)\right) \geq \min \left\{\alpha_{1}, \frac{1}{2} \alpha_{n}\right\} \pi$ by an explicit construction. Consider

$$
\begin{aligned}
& M_{1}=\mathbb{I}^{n}, \quad t_{1}=\mathbf{0}, \\
& M_{2}=\left(\begin{array}{cccc} 
& & 0 \\
& \mathbb{I}^{n-1} & \vdots \\
-1 & \ldots & -1 & 1
\end{array}\right), t_{2}=\left(\begin{array}{c}
0 \\
\vdots \\
0 \\
r
\end{array}\right) .
\end{aligned}
$$


Thus $M_{1}\left(\triangle^{n}(r)\right)$ has vertices $(0, \ldots, 0),(r, 0, \ldots, 0)$, $\ldots,(0, \ldots, 0, r)$, and likewise $M_{2}\left(\triangle^{n}(r)\right)$ has vertices $(r, 0, \ldots, 0), \ldots,(0, \ldots, 0, r),(0, \ldots, 0,2 r)$. It is easy to check that the two simplices are disjoint as they share a common face with vertices $(r, 0, \ldots, 0)$, $\ldots,(0, \ldots, 0, r)$. If $r \leq \alpha_{1}$ and $2 r \leq \alpha_{n}$, both simplices are contained in $P\left(\alpha_{1}, \ldots, \alpha_{n}\right)$. Thus there exists a packing of $\triangle^{n}(1)$ by two copies of $\triangle^{n}(r)$ for $r=\min \left\{\alpha_{1}, \frac{1}{2} \alpha_{n}\right\}$, and the claim about $s_{2}$ follows.

Corollary 3.11. If $\alpha_{1} \leq \alpha_{2} \leq \cdots \leq \alpha_{n}$ then

$$
g_{2}\left(E\left(\sqrt{\alpha_{1}}, \ldots, \sqrt{\alpha_{n}}\right)\right)=\min \left\{\alpha_{1}, \frac{1}{2} \alpha_{n}\right\} \pi .
$$

Proof. Since $E\left(\sqrt{\alpha_{1}}, \ldots, \sqrt{\alpha_{n}}\right) \subset Z\left(\sqrt{\alpha_{1}}, \ldots, \sqrt{\alpha_{n}}\right)$ and $E\left(\sqrt{\alpha_{1}}, \ldots, \sqrt{\alpha_{n}}\right) \subset B^{2 n}\left(\sqrt{\alpha_{n}}\right)$, monotonicity in Definition 2.4 implies $g_{2}\left(E\left(\sqrt{\alpha_{1}}, \ldots, \sqrt{\alpha_{n}}\right)\right) \leq$ $\min \left\{\alpha_{1}, \frac{1}{2} \alpha_{n}\right\} \pi$. Then since

$$
\begin{aligned}
s_{k}\left(P\left(\alpha_{1}, \ldots, \alpha_{n}\right)\right) & \leq g_{k}\left(P\left(\alpha_{1}, \ldots, \alpha_{n}\right)\right) \\
& =g_{k}\left(E\left(\sqrt{\alpha_{1}}, \ldots, \sqrt{\alpha_{n}}\right)\right),
\end{aligned}
$$

the result follows from Theorem 3.10.

\section{PACKING ANALOGUES OF SURFACE PRODUCTS}

As mentioned in Section $1, \Sigma\left(\alpha_{1}, \ldots, \alpha_{n}\right)$ is an $\mathcal{M}_{\mathbb{Z}^{-}}$ analogue of the polydisc with radii $\alpha_{1}, \ldots, \alpha_{n}$. Analogous to results from Section 3, it is possible to exactly compute the simplex widths $s_{k}$ of $\Sigma\left(\alpha_{1}, \ldots, \alpha_{n}\right)$ for "small" $k$. This theorem can be viewed as an easy consequence of a simplex analogue of McDuff and Polterovich's result [1994] that the $2 n$-dimensional polydisc $D^{2}(\alpha) \times \cdots \times D^{2}(\alpha)$ can be fully packed by $n$ ! balls. Some explicit constructions for such packings for dimension 4 are given in [Traynor 1995]. The interesting idea behind the incomplete attempt in [Krouglikov 1994] for explicit full packings of the $2 n$-dimensional polydisc would have led to full simplex $n$ !-packings of $\Sigma(1, \ldots, 1)$. The proof of the following theorem gives a full packing construction of the $2 n$-dimensional polydisc using ideas from symmetry groups.

Theorem 4.1. If $\alpha_{1} \leq \cdots \leq \alpha_{n}$ then

$$
s_{k}\left(\Sigma\left(\alpha_{1}, \ldots, \alpha_{n}\right)\right)=\alpha_{1} \pi \quad \text { for } \quad 1 \leq k \leq n ! .
$$

Proof. By the inclusion $\Sigma\left(\alpha_{1}, \ldots, \alpha_{n}\right) \subset Y^{2 n}\left(\alpha_{1}\right)$, monotonicity in Definition 2.4 implies that

$$
s_{k}\left(\Sigma\left(\alpha_{1}, \ldots, \alpha_{n}\right)\right) \leq \pi \alpha_{1},
$$

for all $k$. Note that for $k \leq n$ !,

$$
s_{k}\left(\Sigma\left(\alpha_{1}, \ldots, \alpha_{n}\right)\right) \geq s_{n !}\left(\Sigma\left(\alpha_{1}, \ldots, \alpha_{n}\right)\right)
$$

and that, by monotonicity,

$$
s_{n !}\left(\Sigma\left(\alpha_{1}, \ldots, \alpha_{n}\right)\right) \geq s_{n !}\left(\Sigma^{2 n}\left(\alpha_{1}\right)\right) .
$$

Thus it suffices to prove that $s_{n !}\left(\Sigma^{2 n}\left(\alpha_{1}\right)\right)=\pi \alpha_{1}$. This will be demonstrated by applying ideas from symmetry groups to construct an explicit full packing of the cube. (See, for example, [Benson and Grove 1971] for background on symmetry groups and fundamental regions.) The symmetry group of the $n$-dimensional cube centered at the origin with the $2^{n}$ vertices $\left( \pm \alpha_{1}, \ldots, \pm \alpha_{1}\right)$ consists of the $n \times n$ signed permutation matrices. A fundamental region for the symmetry group of this $n$-dimensional cube is

$$
F=\left(\begin{array}{ccccc}
1 & 0 & 0 & \cdots & 0 \\
1 & 1 & 0 & \cdots & 0 \\
\vdots & \vdots & \cdots & \cdots & \vdots \\
1 & 1 & 1 & 1 & 1
\end{array}\right)\left(\triangle^{n}\left(\alpha_{1}\right)\right)
$$

Moreover, the volume of $F$ is $\alpha_{1}^{n} / n$ !. The orbit of $F$ under the subgroup comprised of the $n$ ! permutation matrices gives a full packing of $\square\left(\alpha_{1}, \ldots, \alpha_{1}\right)$. It follows that

$$
s_{n !}\left(\mathbb{T}^{n} \times \square\left(\alpha_{1}, \ldots, \alpha_{1}\right)\right)=\pi \alpha_{1} .
$$

In dimension 4 , it is possible to extend the result of Theorem 4.1.

Theorem 4.2. If $1 \leq \alpha$ then

$$
s_{3}(\Sigma(1, \alpha))=\min \left\{1, \frac{1}{3}(1+\alpha)\right\} \pi .
$$

By conformality, Theorem 4.2 is equivalent to the statement that if $\alpha_{1} \leq \alpha_{2}$ then $s_{3}\left(\Sigma\left(\alpha_{1}, \alpha_{2}\right)\right)=$ $\min \left\{\alpha_{1}, \frac{1}{3}\left(\alpha_{1}+\alpha_{2}\right)\right\} \pi$. The proof of Theorem 4.2 illustrates the technicalities involved when proving simplex widths "by hand". We give an overview of the proof. If $\alpha \geq 2$, it is not hard to show that $s_{3}(\Sigma(1, \alpha))=\pi$. If $\alpha<2$, an application of Proposition 3.9 will show that when $r>\frac{1}{3}(1+\alpha)$ there do not exist two triangles of the same shape in a packing of $\square\left(\alpha_{1}, \alpha_{2}\right)$ by images of $\triangle^{2}(r)$. By generalizing Proposition 3.9, it will then be shown that at most two different shaped triangles can exist in the packing. Thus a 3 -packing is impossible. 
Proposition 4.3 (Different-shape noncompatibility). For $k=1,2$, let $T_{k}=M_{k}(\triangle(r)), M_{k} \in \mathrm{GL}(n, \mathbb{Z})$, and let $\tau_{k}$ be the convex hull of $z_{j}^{k}$ :

$$
\tau_{k}=\left\{\sum_{i=1}^{m_{k}} t_{i} z_{i}^{k} \mid t_{i} \geq 0 \text { and } \sum_{i=1}^{m_{k}} t_{i}=1\right\} .
$$

If

$$
\left(T_{1}+z_{i}^{1}\right) \cap\left(T_{2}+z_{j}^{2}\right) \neq \varnothing
$$

for all $i=1, \ldots, m_{1}$ and $j=1, \ldots, m_{2}$, then

$$
\left(T_{1}+\alpha_{1}\right) \cap\left(T_{2}+\alpha_{2}\right) \neq \varnothing
$$

for all $\alpha_{1} \in \tau_{1}$ and $\alpha_{2} \in \tau_{2}$.

Proof. For $k=1,2$, let $\alpha_{k}=\sum_{i=1}^{m_{k}} t_{i}^{k} z_{i}^{k} \in \tau_{k}$ be arbitrary. By hypothesis, there exists

$$
p_{i j} \in\left(T_{1}+z_{i}^{1}\right) \cap\left(T_{2}+z_{j}^{2}\right) .
$$

For a fixed $j$, Lemma 3.8 and the convexity of $T_{2}+z_{j}^{2}$ imply

$$
\sum_{i=1}^{m_{1}} t_{i}^{1} p_{i j} \in\left(T_{1}+\alpha_{1}\right) \cap\left(T_{2}+z_{j}^{2}\right) .
$$

Furthermore, by Lemma 3.8 and the fact that $T_{1}+\alpha_{1}$ is convex,

$$
\sum_{j=1}^{m_{2}} t_{j}^{2}\left(\sum_{i=1}^{m_{1}} t_{i}^{1} p_{i j}\right) \in\left(T_{1}+\alpha_{1}\right) \cap\left(T_{2}+\alpha_{2}\right) .
$$

Hence, $\left(T_{1}+\alpha_{1}\right) \cap\left(T_{2}+\alpha_{2}\right) \neq \varnothing$ for all $\alpha_{1} \in \tau_{1}$ and $\alpha_{2} \in \tau_{2}$.

Proof of Theorem 4.2. Since $\Sigma(1, \alpha) \subset Y(1)$, monotonicity implies $s_{k}(\Sigma(1, \alpha)) \leq \pi$, for all $k$. An explicit construction shows this is optimal when $\alpha \geq 2$. Namely, consider the packing of $\square(1, \alpha)$ by $\triangle^{2}(1)$ given by

$$
\begin{array}{ll}
M_{1}=\left(\begin{array}{ll}
1 & 0 \\
0 & 1
\end{array}\right), & t_{1}=\left(\begin{array}{l}
0 \\
0
\end{array}\right), \\
M_{2}=\left(\begin{array}{rr}
-1 & 0 \\
0 & -1
\end{array}\right), & t_{2}=\left(\begin{array}{l}
1 \\
1
\end{array}\right), \\
M_{3}=\left(\begin{array}{rr}
-1 & 0 \\
0 & -1
\end{array}\right), & t_{3}=\left(\begin{array}{l}
1 \\
2
\end{array}\right) .
\end{array}
$$

See Figure 4.4 (which repeats Figure 1.19 for convenience).
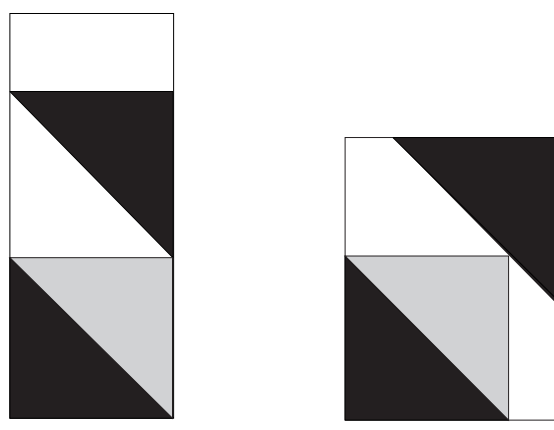

FIGURE 4.4. Optimal simplex packing configurations with 3 objects when $\alpha_{2} \geq 2 \alpha_{1}$ and when $\alpha_{2} \leq 2 \alpha_{1}$.

Next consider the case $\alpha<2$. If $r=\frac{1}{3}(1+\alpha)$ then

$$
\begin{array}{ll}
M_{1}=\left(\begin{array}{ll}
1 & 0 \\
0 & 1
\end{array}\right), & t_{1}=\left(\begin{array}{l}
0 \\
0
\end{array}\right), \\
M_{2}=\left(\begin{array}{rr}
-1 & 0 \\
0 & -1
\end{array}\right), & t_{2}=\left(\begin{array}{l}
r \\
r
\end{array}\right), \\
M_{3}=\left(\begin{array}{rr}
-1 & 0 \\
0 & -1
\end{array}\right), & t_{3}=\left(\begin{array}{l}
1 \\
\alpha
\end{array}\right),
\end{array}
$$

proves that $s_{3}(\Sigma(1, \alpha)) \geq \frac{1}{3}(1+\alpha)$. See Figure 4.4 . To show that $r=\frac{1}{3}(1+\alpha)$ is optimal, suppose that there is a simplex 3-packing of $\square(1, \alpha)$ with $\triangle^{2}(r)$ where $r>\frac{1}{3}(1+\alpha)$. Note that $r>\frac{1}{3}(1+\alpha)>$ $\frac{1}{3}\left(\frac{1}{2} \alpha+\alpha\right)=\frac{1}{2} \alpha$. Thus an argument as in the proof of Lemma 3.4 proves that the vertices of $M\left(\triangle^{2}(r)\right)+$ $t$ must coincide with 3 vertices of a translated cube of size $r$. In particular, $[M]$ must be either $\left[\left(\begin{array}{ll}1 & 0 \\ 0 & 1\end{array}\right)\right]$, $\left[\left(\begin{array}{cc}-1 & 0 \\ 0 & 1\end{array}\right)\right],\left[\left(\begin{array}{cc}1 & 0 \\ 0 & -1\end{array}\right)\right]$, or $\left[\left(\begin{array}{cc}-1 & 0 \\ 0 & -1\end{array}\right)\right]$. Without loss of generality, it can be assumed that any simplex 3packing of $\square(1, \alpha)$ has $\left[M_{1}\right]=\left[\left(\begin{array}{ll}1 & 0 \\ 0 & 1\end{array}\right)\right]$. This follows since given any packing of $\square(1, \alpha)$, there exists a compositions of reflections and translations that map $\square(1, \alpha)$ to itself and a given triangle in the packing to a translate of $T:=\left(\begin{array}{ll}1 & 0 \\ 0 & 1\end{array}\right) \triangle(r)$. Proposition 3.9 will now be used to show that there do not exist two triangles with this shape in a packing. The translation set $\tau$ for $T$ is the rectangle with vertices $t_{1}=(0,0), t_{2}=(1-r, 0), t_{3}=(1-r, \alpha-r)$, $t_{4}=(0, \alpha-r)$. Note that if $r>\frac{1}{3}(1+\alpha)$, then $1-r<1-\left(\frac{1}{3}(1+\alpha)\right)=\frac{1}{3}(2-\alpha)$, and

$$
\alpha-r<\alpha-\left(\frac{1}{3}(1+\alpha)\right)=\frac{1}{3}(2 \alpha-1) .
$$

Then $\left(\frac{1}{3}(2-\alpha), \frac{1}{3}(2 \alpha-1)\right) \in T+t_{i}$ when $i=1,2,3,4$. Thus, there exists at most one such triangle in a packing. The arguments above also imply there exists at most one embedding of each of the other 
shaped triangles in a packing of $\square(1, \alpha)$. Since there cannot be two triangles of the same shape, it can be assumed that $\left[M_{2}\right]=\left[\left(\begin{array}{cc}-1 & 0 \\ 0 & 1\end{array}\right)\right],\left[\left(\begin{array}{cc}1 & 0 \\ 0 & -1\end{array}\right)\right]$, or $\left[\left(\begin{array}{cc}-1 & 0 \\ 0 & -1\end{array}\right)\right]$. Consider the first option: let $S=\left(\begin{array}{cc}-1 & 0 \\ 0 & 1\end{array}\right) \triangle(r)$. Notice that $T$ and $S$ have the same translation set $\tau$ as described above. An application of Proposition 4.3 will show that $(T+\alpha) \cap(S+\beta) \neq \varnothing$ for $\alpha, \beta \in \tau$. Let $T_{i}=T+t_{i}$ and $S_{i}=S+t_{i}$. To show that $T_{i} \cap S_{j} \neq \varnothing$ for all $i, j \in\{1, \ldots, 4\}$, it suffices to show that the edges of the closures of $T_{i}$ and $S_{j}$ intersect transversally at a nonvertex point $x_{i j}$. Direct calculations show such intersections occur at $x_{11}=$ $\left(\frac{1}{2} r, \frac{1}{2} r\right), x_{12}=\left(\frac{1}{2}, r-\frac{1}{2}\right), x_{13}=(2 r-\alpha, \alpha-r) x_{14}=$ $(2 r-\alpha, \alpha-r), x_{21}=(1-r, 1-r), x_{22}=\left(1-\frac{1}{2} r, \frac{1}{2} r\right)$, $x_{23}=(r+1-\alpha, \alpha-r), x_{24}=(1-r, \alpha-r)$. Notice, for instance, that the intersection $x_{12}$ is guaranteed as long as $r>\frac{1}{2}$ whereas the existence of $x_{13}$ requires $r>\frac{1}{3}(1+\alpha)$. By reflections and translations, the existence of $x_{24}, x_{14}, x_{22}$, and $x_{21}$ implies the existence of $x_{31}, x_{32}, x_{33}$, and $x_{34}$. Similarly, the existence of $x_{23}, x_{13}, x_{12}$, and $x_{22}$ implies the existence of $x_{41}, x_{42}, x_{43}$ and $x_{44}$. Thus, there do not exist disjoint translates of $T$ and $S$ in $\square(1, \alpha)$. A similar argument shows that it is not possible to disjointly embed translates of $T$ and $R=\left(\begin{array}{cc}1 & 0 \\ 0 & -1\end{array}\right) \triangle(r)$. Thus if $1 \leq \alpha<2$ and $r>\frac{1}{3}(1+\alpha)$, there exist at most two triangles in a packing of $\square(1, \alpha)$.

There are many interesting questions beyond calculating the simplex width capacities of a fixed symplectic manifold. For example, it would be interesting to understand the possible effect of removing subsets of volume 0 on the simplex widths. In the following, some results and conjectures are given about the possible beginnings of the "simplex capacity spectrum" for $\mathbb{T}^{2} \times U$ where $U$ is an open subset of $\square(1)$.

Conjecture 4.5. Consider an open, contractible set $U \subset \square(1)$. Let $s_{1}=r_{1} \pi, s_{2}=r_{2} \pi, s_{3}=r_{3} \pi$ be the first three simplex widths of $\mathbb{T}^{2} \times U$. Then $r_{1}, r_{2}, r_{3}$ must satisfy $0<r_{1} \leq 1, \quad \frac{1}{2} r_{1} \leq r_{2} \leq r_{1}$, and

$$
\frac{1}{2} r_{1} \leq r_{3} \leq \min \left\{r_{2}, \max \left\{2-\left(r_{1}+r_{2}\right), \frac{1}{3}\left(r_{1}+r_{2}\right)\right\}\right\} .
$$

Conversely, if $r_{1}, r_{2}, r_{3}$ are chosen arbitrarily so that they satisfy these inequalities, there exists a $U$ and a volume-preserving diffeomorphism between $\mathbb{T}^{2} \times U$ and $\mathbb{T}^{2} \times \square(1)$ so that $s_{1}=r_{1} \pi, s_{2}=r_{2} \pi, s_{3}=r_{3} \pi$ are the first three simplex widths of $\mathbb{T}^{2} \times U$.
Portions of this conjecture can be proved. First consider an open $U \subset \square(1)$. All the inequalities except $r_{3} \leq \max \left\{2-\left(r_{1}+r_{2}\right), \frac{1}{3}\left(r_{1}+r_{2}\right)\right\}$ are easy to prove.

Now suppose $r_{1}, r_{2}, r_{3}$ are chosen so that they satisfy the stated inequalities. The idea will be to construct $U$ by removing line segments from $\square(1)$ in such a way that $U$ is still open and contractible. In all cases, conjectured optimal packings can be constructed using only right, isosceles triangles.

The construction of $U$ depends on the quantity $\max \left\{2-\left(r_{1}+r_{2}\right), \frac{1}{3}\left(r_{1}+r_{2}\right)\right\}$. First suppose that this quantity equals $2-\left(r_{1}+r_{2}\right)$. In this case, $r_{1}, r_{2}$ are small enough that it is possible to construct packings so that that there exist disjoint triangles from the optimal 1, 2, and 3 simplex packings. The upper bound for $r_{3}$ comes from the fact that in order for these triangles to be disjoint, the sum of the "diagonals" of the three disjoint right isosceles triangles, namely $\frac{1}{2} r_{1} \sqrt{2}+\frac{1}{2} r_{2} \sqrt{2}+\frac{1}{2} r_{3} \sqrt{2}$ must be less than or equal to $\sqrt{2}$, the length of the diagonal of $\square(1)$. See the figures in Figure 4.6(a). Construct $U \subset \square(1)$ as follows. Delete paths from the boundaries of the three triangles $\left(\begin{array}{ll}1 & 0 \\ 0 & 1\end{array}\right) \triangle\left(r_{1}\right),\left(\begin{array}{cc}-1 & 0 \\ 0 & -1\end{array}\right) \triangle\left(r_{2}\right)+\left(\begin{array}{l}r_{2} \\ r_{1}\end{array}\right)$, $\left(\begin{array}{cc}-1 & 0 \\ 0 & -1\end{array}\right) \triangle\left(r_{3}\right)+\left(\begin{array}{l}1 \\ 1\end{array}\right)$ such that each path has one endpoint on the boundary of $\square(1)$ and traces out much of the boundary without disconnecting $\square(1)$. In the complement of the above three triangles, delete a sufficient number of line segments to guarantee that no larger triangles can be embedded. The first three simplex widths of $\mathbb{T}^{2} \times U$ appear to be $r_{1} \pi, r_{2} \pi$, and $r_{3} \pi$.

Next suppose that $\max \left\{2-\left(r_{1}+r_{2}\right), \frac{1}{3}\left(r_{1}+r_{2}\right)\right\}=$ $\frac{1}{3}\left(r_{1}+r_{2}\right)$. Now $r_{1}, r_{2}$ are so large that it is more efficient to pack 3 simplices inside the images of the optimal 1 and 2-packings. See Figure 4.6(b,c). The upper bound for $r_{3}$ by $\frac{1}{3}\left(r_{1}+r_{2}\right)$ is related to the bound

$$
s_{3}\left(\mathbb{T}^{2} \times \square\left(r_{1}, r_{2}\right)\right) \leq \frac{1}{3}\left(r_{1}+r_{2}\right)
$$

from Theorem 4.2. Construct $U \subset \square(1)$ by deleting lines in the complement of the closures of the images of the triangles $\left(\begin{array}{ll}1 & 0 \\ 0 & 1\end{array}\right) \triangle\left(r_{1}\right)$ and $\left(\begin{array}{cc}-1 & 0 \\ 0 & -1\end{array}\right) \triangle\left(r_{2}\right)+\left(\begin{array}{l}r_{2} \\ r_{1}\end{array}\right)$. See the horizontal lines in Figure $4.6(\mathrm{~b}, \mathrm{c})$, and the second figure in (d). In addition, delete paths from the boundaries of the triangles under the following condition. If $r_{3}=\frac{1}{2} r_{1}$, delete a path along the line $y_{1}+y_{2}=r_{1}$ with one endpoint on the boundary of $\square(1)$ as in 4.6 (b). If $r_{3}>\frac{1}{2} r_{1}$, delete two paths along 

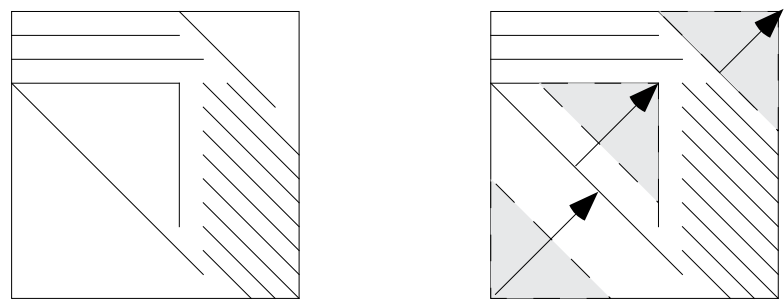

(a)
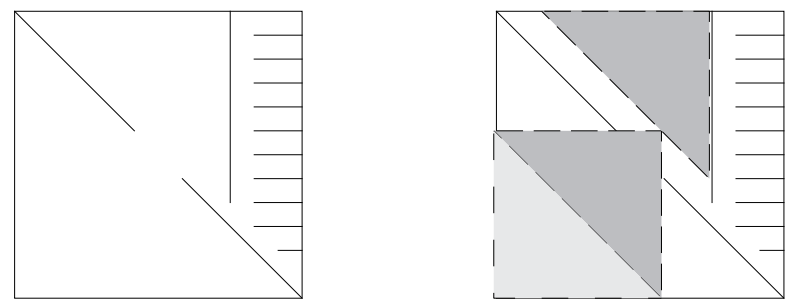

(c)

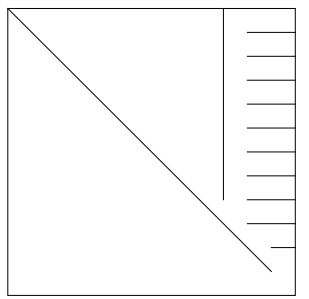

(b)
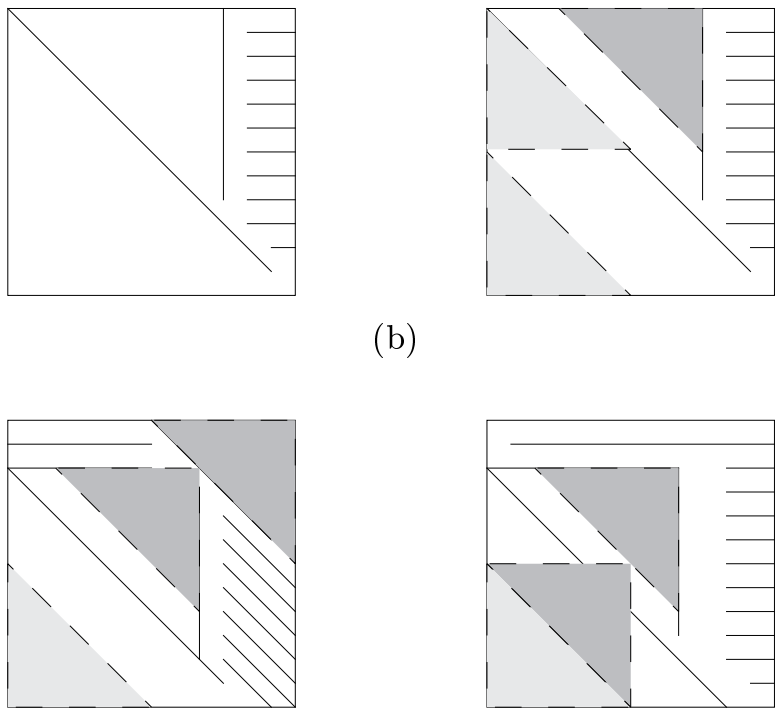

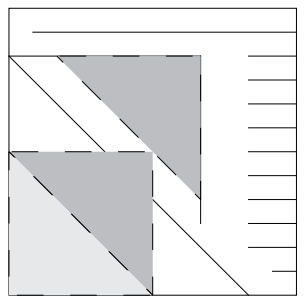

(d)

FIGURE 4.6. (a) Construction of $U$ in the case $\max \left\{2-\left(r_{1}+r_{2}\right), \frac{1}{3}\left(r_{1}+r_{2}\right)\right\}=2-\left(r_{1}+r_{2}\right)$. The arrows denote the "diagonals" of the triangles from believed optimal simplex 1,2,and 3-packings. (b) Construction of $U$ in the case $\max \left\{2-\left(r_{1}+r_{2}\right), \frac{1}{3}\left(r_{1}+r_{2}\right)\right\}=\frac{1}{3}\left(r_{1}+r_{2}\right), r_{3}=\frac{r_{1}}{2}$ together with its believed optimal simplex 3-packing. (c) Construction of $U$ in the case $\max \left\{2-\left(r_{1}+r_{2}\right), \frac{1}{3}\left(r_{1}+r_{2}\right)\right\}=\frac{1}{3}\left(r_{1}+r_{2}\right), r_{3}>\frac{r_{1}}{2}$ together with its believed optimal simplex 3-packing. (d) Different options of the construction of $U$ when $2-\left(r_{1}+r_{2}\right)=\frac{1}{3}\left(r_{1}+r_{2}\right)$.

the line $y_{1}+y_{2}=r_{1}$ from the boundary of $\square(1)$ to the boundary of $\left(\begin{array}{cc}-1 & 0 \\ 0 & -1\end{array}\right) \triangle\left(r_{3}\right)+\left(\begin{array}{c}r_{3} \\ r_{3}\end{array}\right)$ as in $4.6(\mathrm{c})$.

Notice that $2-\left(r_{1}+r_{2}\right)=\frac{1}{3}\left(r_{1}+r_{2}\right)$ if and only if $r_{1}+r_{2}=\frac{3}{2}$. In this case, $U$ can be constructed by either of the two ways above; see Figure 4.6(d).

\section{SIMPLEX PACKING COMPUTER ALGORITHMS}

This section describes the algorithms used to compute the simplex packing widths in Theorems 1.2, $1.8,1.13$, and 1.16. The main idea is to compute the optimal packing of $k$ images of a simplex into an $n$ dimensional polytope by maximizing a linear form over an $(n k+1)$-dimensional "configuration space". This complicated space can easily be decomposed into a large union of (convex) polytopes. For packing $k \leq 12$ simplices in $\triangle^{2}$, or $k \leq 11$ simplices in $\square^{2}$, or $k \leq 8$ simplices in $\square^{3}$, it is feasible to construct and examine all the polytopes.

Despite some shortcuts, our method essentially uses brute force, and the combinatorial explosion in this problem is severe. In dimension 2 , the exact results for $k \leq 8$ were obtained using a simple Mathematica program running on a workstation. By contrast, to reach $k=12$ in dimension 2 and $k=8$ in dimension 3 required specialized programs running on a massively parallel supercomputer. These programs allowed us to perform about $10^{7}$ times more computation with perhaps $10^{4}$ times more storage for intermediate results. Nonetheless, $k=13$ still seems out of reach for the moment, and reaching the "next level" $(k>16$ triangles in a triangle or $k>18$ triangles in a square) is out of the question without some new approach. As described in the last subsection of the paper, however, it is still possible to give estimates for larger $k$ by packing "convex lattice polygons".

\section{Configuration Spaces}

For $1 \leq i \leq k$, let $\triangle_{i}$ be an image of the standard open $n$-simplex $\triangle^{n}$ under an element of $\operatorname{GL}(n, \mathbb{Z})$, and let $S \subset R^{n}$ be the interior of a convex polytope. The configuration space $X\left(\triangle_{1}, \ldots, \triangle_{k}\right)$ of valid packings is the set of points $\left(t_{1}, \ldots, t_{k}, r\right) \in$ $\left(\mathbb{R}^{n}\right)^{k} \times \mathbb{R}^{+}$satisfying

$$
r \triangle_{i}+t_{i} \subseteq S \text { for } 1 \leq i \leq k
$$

and

(5.2)

$$
\left(r \triangle_{i}+t_{i}\right) \cap\left(r \triangle_{j}+t_{j}\right)=\varnothing \quad \text { for } 1 \leq i<j \leq k .
$$


(Compare Definition 3.5.)

Let $\mathcal{D}$ denote the multiset $\left\{\triangle_{1}, \ldots, \triangle_{k}\right\}$. Our goal is to find the largest value of $r$, call it $r(\mathcal{D})$, such that $X\left(\triangle_{1}, \ldots, \triangle_{k}\right) \cap\left(\mathbb{R}^{n k} \times\{r\}\right)$ is nonempty, and then maximize $r(\mathcal{D})$ over all multisets $\mathcal{D}$. Since there always exists a packing for some sufficiently small value of $r$, say $r=\varepsilon$, it is possible to ignore all shapes $\triangle$ such that no translate of $\varepsilon \triangle$ lies in $S$. This leaves finitely many possibilities for each $\triangle_{i}$ and hence finitely many multisets $\mathcal{D}$ to consider.

The set $X\left(\triangle_{1}, \ldots, \triangle_{k}\right)$ has a complicated but finite description in terms of linear inequalities. If $S$ is expressed as an intersection of half-spaces $\left\{y \in \mathbb{R}^{n}\right.$ $a \cdot y<b\}$, where $a \in \mathbb{R}^{n}$ and $b \in \mathbb{R}$, then the condition $r \triangle_{i}+t_{i} \subseteq S$ is captured by the corresponding inequalities

$$
a \cdot t_{i}+c r \leq b, \quad \text { where } c=\sup _{y \in \triangle_{i}} a \cdot y .
$$

Consequently, the set of configurations satisfying (5.1) is a polytope $P \subset \mathbb{R}^{n k} \times \mathbb{R}^{+}$.

Condition (5.2) removes a collection of convex sets from $P$. To see this, first notice that $r \triangle_{i}+t_{i}$ intersects $r \triangle_{j}+t_{j}$ if and only if $t_{j}-t_{i}$ lies in the Minkowski sum

$$
r \triangle_{i}+\left(-r \triangle_{j}\right)=\left\{y_{i}-y_{j} \mid y_{i} \in r \triangle_{i}, y_{j} \in r \triangle_{j}\right\},
$$

which we write as $r\left(\triangle_{i}-\triangle_{j}\right)$. Define the forbidden set

$$
F_{i j}=\left\{(y, r) \in \mathbb{R}^{n} \times \mathbb{R}^{+} \mid y \in r\left(\triangle_{i}-\triangle_{j}\right)\right\} .
$$

Geometrically, $F_{i j}$ is a cone over the open set $\triangle_{i}-\triangle_{j}$ (without its vertex). By the remarks above, the configuration space is obtained from $P$ by omitting all points $\left(t_{1}, \ldots, t_{k}, r\right)$ such that $t_{j}-t_{i} \in F_{i j}$, where $1 \leq i<j \leq k$. Let $\pi_{i j}: \mathbb{R}^{n k} \times \mathbb{R}^{+} \rightarrow \mathbb{R}^{n} \times \mathbb{R}^{+}$be the linear map $\left(t_{1}, \ldots, t_{k}, r\right) \mapsto\left(t_{j}-t_{i}, r\right)$. Then

$$
X\left(\triangle_{1}, \ldots, \triangle_{k}\right)=P \backslash \bigcup_{i<j} \pi_{i j}^{-1}\left(F_{i j}\right) .
$$

This leads to a decomposition of $X\left(\triangle_{1}, \ldots, \triangle_{k}\right)$ into finitely many polytopes. Write $\left(\mathbb{R}^{n} \times \mathbb{R}^{+}\right) \backslash F_{i j}$ as a union of $f_{i j}$ convex polyhedra $A_{i j}^{1} \cup \cdots \cup A_{i j}^{f_{i j}}$, where $f_{i j}$ is the number of facets of $\triangle_{i}-\triangle_{j}$. Figure 5.3 , right, illustrates the construction for dimension $n=2$. Then

$$
X\left(\triangle_{1}, \ldots, \triangle_{k}\right)=\bigcup_{\sigma}\left(P \cap \bigcap_{i<j} \pi_{i j}^{-1}\left(A_{i j}^{\sigma(i, j)}\right)\right),
$$
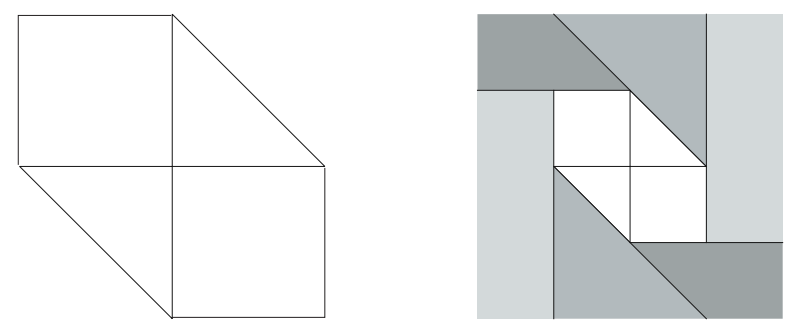

FIGURE 5.3. Left: The Minkowski sum $\triangle_{i}+\left(-\triangle_{j}\right)$ is the interior of this hexagon when $\triangle_{i}$ and $\triangle_{j}$ are the images of the standard simplex $\triangle^{2}$ under $M=\mathbb{I}$. Right: The cross section at $r=\frac{1}{2}$ of the complement of the forbidden set (when $\triangle_{i}=\triangle_{j}=\triangle^{2}$ ) subdivided into 6 closed polyhedra with disjoint interiors. Each polyhedron $A_{i j}^{l}$ is the intersection of two half-spaces.

where the union is over all functions $\sigma:\{(i, j) \mid 1 \leq$ $i<j \leq k\} \rightarrow\left\{1, \ldots, f_{i j}\right\}$. For each such $\sigma$, the intersection $P \cap \bigcap_{i<j} \pi_{i j}^{-1}\left(A_{i j}^{\sigma(i, j)}\right)$ is a polytope $X_{\sigma}$ defined by finitely many linear inequalities. Hence, by solving linear programs, it is possible to maximize $r$ over each of the $\prod_{i<j} f_{i j}$ polytopes $X_{\sigma}$, and the largest of these maxima is $r(\mathcal{D})$.

Remark 5.4. If $S$ is the simplex $\triangle^{n}(1)$ or the cube $\square^{n}(1)$ then the calculation of $r(\mathcal{D})$ can be carried out exactly. In these cases the closures of $S$ and $\triangle_{i}-\triangle_{j}$ are polytopes with integer vertices, and hence each of the inequalities that define the polyhedra $P$ and $A_{i j}^{l}$ can be written with integer coefficients. Therefore the linear programs can be solved using exact rational arithmetic. It follows, as mentioned in Section 1, that the maximum value of $r$ is rational, and hence that the simplex packing widths are rational multiples of $\pi$.

\section{Practical Algorithms}

The algorithm just described is not practical as it stands, because the number $\prod_{i<j} f_{i j}$ of polytopes $X_{\sigma}$ can be astronomical - on the order of $6^{k(k-1) / 2}$ in dimension $n=2$ - and the number of multisets $\mathcal{D}$ is exponential in $k$. To make the algorithm practical even for modest values of $k$, we employ several ideas to reduce the amount of calculation needed. Nonetheless, the time and space requirements of the algorithm remain exponential in $k^{2}$, whether one uses the simplex algorithm for solving linear programs, as we do, or a theoretically faster method. 
The goal of the improvements is merely to bring a few more cases within reach.

We accelerate the calculation of $r(\mathcal{D})$ by organizing the functions $\sigma$ in a "branch-and-bound" algorithm. First we find a packing that is likely to be optimal, either by hand or by the "lattice polygon" method described in the last subsection of this paper. Let $r_{0}$ be the value of $r$ for this packing. Then we search for functions $\sigma$ such that the maximum value $r(\sigma)$ attained by $r$ on $X_{\sigma}$ exceeds $r_{0}$. Starting with all values $\sigma(i, j)$ undefined, we choose some ordering on the pairs $(i, j)$ with $1 \leq i<j \leq k$, and successively fill in the entries $\sigma(i, j)$ in all possible ways. This amounts to searching a tree of height $\left(\begin{array}{l}k \\ 2\end{array}\right)$ whose nodes on level $l$ are the functions $\rho$ from the first $l$ pairs $(i, j)$ to integers $\rho(i, j) \in\left[1, f_{i j}\right]$, with edges between nodes denoting function extensions.

The key observation is that a function $\rho$ yields an upper bound on $r(\sigma)$ for all functions $\sigma$ that extend $\rho$. Let $X_{\rho}$ be the polytope

$$
P \cap \bigcap \pi_{i j}^{-1}\left(A_{i j}^{\rho(i, j)}\right),
$$

where the intersection is taken over the pairs $(i, j)$ such that $\rho(i, j)$ is defined, and let $r(\rho)$ be the maximum value attained by $r$ on $X_{\rho}$. If $\sigma$ extends $\rho$, then $X_{\sigma} \subseteq X_{\rho}$ and hence $r(\sigma) \leq r(\rho)$. We can compute $r(\rho)$ by solving a linear program. If $r(\rho) \leq r_{0}$, then we can safely discard $\rho$ and ignore all functions $\sigma$ that extend it. Thus we can search the tree depth-first, computing $r(\rho)$ at each node and backtracking whenever $r(\rho) \leq r_{0}$. If we ever reach a leaf $\sigma$ with $r(\sigma)>r_{0}$, we can revise our value for $r_{0}$ and make the remainder of the search more efficient. In practice, however, we search the tree breadth-first; although it requires more memory, it allows us to perform many computations in parallel. If $r_{0}$ is indeed optimal and the entries $\rho(i, j)$ are considered in an intelligent order, the search can usually prove that $r(\mathcal{D}) \leq r_{0}$ while exploring only a tiny fraction of the tree.

Similarly, there is no need to prove that $r(\mathcal{D}) \leq r_{0}$ for every multiset of shapes $\mathcal{D}$. It suffices to find a blocking set of multisets $\mathcal{E}_{1}, \ldots, \mathcal{E}_{N}$ such that every multiset $\mathcal{D}$ contains some $\mathcal{E}_{i}$ and $r\left(\mathcal{E}_{i}\right) \leq r_{0}$ for each $i$. We use several different techniques to find efficient blocking sets, some automated and some requiring human intervention. Typically, we divide the set of available shapes $\triangle$ into a large set $\mathcal{A}$ of "awkward" shapes that cannot be packed efficiently and a small set $\mathcal{B}$ of "basic" shapes that are more common in efficient packings. For instance, when packing the triangle $\triangle^{2}$, the set $\mathcal{B}$ might consist only of the shape $\triangle^{2}$ itself. We then inductively enumerate all multisets $\mathcal{D}$ of shapes from $\mathcal{A}$ such that $r(\mathcal{D})>r_{0}$, starting with the empty multiset and successively adding elements of $\mathcal{A}$ in all possible ways. Call a multiset $\mathcal{D}$ live if $r(\mathcal{D})>r_{0}$ and dead otherwise. By not considering any multisets of cardinality $i$ until we have found all live multisets $\mathcal{E}$ of cardinality $i-1$, we avoid computing $r(\mathcal{D})$ for any multiset $\mathcal{D}$ that contains a dead multiset $\mathcal{E}$. We save for each live multiset $\mathcal{E}$ the functions $\sigma$ such that $r(\sigma)>r_{0}$, to avoid repeating work when considering $\mathcal{D}=\mathcal{E} \cup\left\{\triangle_{i}\right\}$. At the end of this process, we can add all minimal dead multisets $\mathcal{E}$ over $\mathcal{A}$ to the blocking set we are constructing. The remaining task, which is more difficult, is to prove that none of the live multisets $\mathcal{D}$ of awkward shapes can be extended to a live multiset $\mathcal{C}$ of cardinality $k$ by adding basic shapes. We usually treat each multiset $\mathcal{D}$ as a separate subproblem, but sometimes a partial result for one subproblem offers an efficient route to a partial result for another, and this is one place where human insight has been useful.

We have three further ways to save work. First, when $S$ has symmetries in $\operatorname{GL}(n, \mathbb{Z})$, this group of symmetries permutes the multisets of shapes, and we need to compute $r(\mathcal{D})$ for at most one multiset $\mathcal{D}$ in each orbit. Second, if $\mathcal{D}=\left\{\triangle_{1}, \ldots, \triangle_{k}\right\}$ contains a shape with multiplicity $\geq 2$, say $\triangle_{i}=\triangle_{j}$, then interchanging the positions of $\triangle_{i}$ and $\triangle_{j}$ gives an equivalent packing. Geometrically, a product of symmetric groups acts on the configuration space $X\left(\triangle_{1}, \ldots, \triangle_{k}\right)$, and it suffices to consider polytopes $X_{\rho}$ that cover a fundamental domain for this action. Third, and most important, the optimum value $r(\mathcal{D})$ is always achieved by $r(\sigma)$ for some polytope $X_{\sigma}$ of full rank. For if we shrink an optimal packing slightly, setting $r=r(\mathcal{D})-\varepsilon$ and preserving the centroid of each simplex in the packing, then all the simplices can move freely and independently; we see $n k+1$ degrees of freedom. Therefore we can discard any intermediate polytope $X_{\rho}$ that is not of full rank. 


\section{Estimates from Convex Lattice Polygons}

We can use the computer to make informed conjectures about two-dimensional packings for larger values of $k$. In the two-dimensional packings that are known to be optimal, many of the triangles abut edge-to-edge to form blocks, which are scaled translates of convex lattice polygons; see Figures 1.4(c) and 1.14. In fact, each packing that is known to be optimal consists of at most three blocks. This observation suggests that we look for the best packings of the region $S$ by a small number of blocks. A simple inductive argument using Pick's theorem shows that every lattice polygon can be triangulated by translates of $\mathrm{GL}(2, \mathbb{Z})$-images of $\triangle^{2}$, so each packing by blocks gives rise to a simplex packing. (See, for example, [Coxeter 1961], for background on Pick's theorem.) The estimates in Theorems 1.8 and 1.16 were obtained by this lattice polygon approach. Unfortunately, for the case of 16 triangles in a square we found a packing of 7 blocks that improves upon the best packing of 6 or fewer, which dampens any hope for a strong bound on the number of blocks in an optimal packing. Figure 5.5 illustrates the best block packings found by our programs, excluding trivial cases. We conjecture that these packings yield optimal simplex packings.

\section{ACKNOWLEDGEMENTS}

This study was initiated by a comment of Ya. Eliashberg. We thank MSRI for its support during 199697, when Maley was a member of the program on combinatorics and Traynor was a member of the program on low dimensional topology. In addition, we thank Paul Biran for helpful comments.

\section{REFERENCES}

[Benson and Grove 1971] C. T. Benson and L. C. Grove, Finite reflection groups, Bogden \& Quigley, Tarrytown-on-Hudson, NY, 1971. 2nd ed., Springer, New York, 1985.

[Biran 1997] P. Biran, "Symplectic packing in dimension 4", Geom. Funct. Anal. 7:3 (1997), 420-437.

[Biran 1999a] P. Biran, "Constructing new ample divisors out of old ones", Duke Math. J. 98:1 (1999), 113-135.
[Biran 1999b] P. Biran, "A stability property of symplectic packing", Invent. Math. 136:1 (1999), 123-155.

[Conway and Sloane 1988] J. H. Conway and N. J. A. Sloane, Sphere packings, lattices and groups, Grundlehren der Math. Wissenschaften 290, Springer, New York, 1988.

[Coxeter 1961] H. S. M. Coxeter, Introduction to geometry, Wiley, New York, 1961. 2nd edition, 1969; reprinted 1989 in the Wiley Classics Library.

[Ekeland and Hofer 1989] I. Ekeland and H. Hofer, "Symplectic topology and Hamiltonian dynamics", Math. Z. 200:3 (1989), 355-378.

[Giroux 1994] E. Giroux, "Une structure de contact, même tendue, est plus ou moins tordue", Ann. Sci. École Norm. Sup. (4) 27:6 (1994), 697-705.

[Gromov 1985] M. Gromov, "Pseudoholomorphic curves in symplectic manifolds", Invent. Math. 82:2 (1985), 307-347.

[Hofer and Zehnder 1994] H. Hofer and E. Zehnder, Symplectic invariants and Hamiltonian dynamics, Birkhäuser Advanced Texts, Birkhäuser, Basel, 1994.

[Krouglikov 1994] B. Krouglikov, "Note on symplectic packing constructions", preprint, 1994.

[Lalonde and Sikorav 1991] F. Lalonde and J.-C. Sikorav, "Sous-variétés lagrangiennes et lagrangiennes exactes des fibrés cotangents", Comment. Math. Helv. 66:1 (1991), 18-33.

[Mastrangeli 1997] J. Mastrangeli, Symplectic packings of cotangent bundles of tori, Ph.D. thesis, Bryn Mawr College, Bryn Mawr, PA, 1997.

[McDuff and Polterovich 1994] D. McDuff and L. Polterovich, "Symplectic packings and algebraic geometry", Invent. Math. 115:3 (1994), 405-434. With an appendix by Yael Karshon.

[McDuff and Salamon 1995] D. McDuff and D. Salamon, Introduction to symplectic topology, Oxford Science Publications, Oxford University Press, New York, 1995. 2nd edition, 1998.

[Melissen 1997] H. Melissen, Packing and covering with circles, Ph.D. thesis, Proefschrift Universiteit Utrecht, The Netherlands, 1997.

[Nagata 1959] M. Nagata, "On the 14-th problem of Hilbert", Amer. J. Math. 81 (1959), 766-772.

[Sikorav 1989] J.-C. Sikorav, "Rigidité symplectique dans le cotangent de $T^{n}$ ", Duke Math. J. 59:3 (1989), 759-763. 

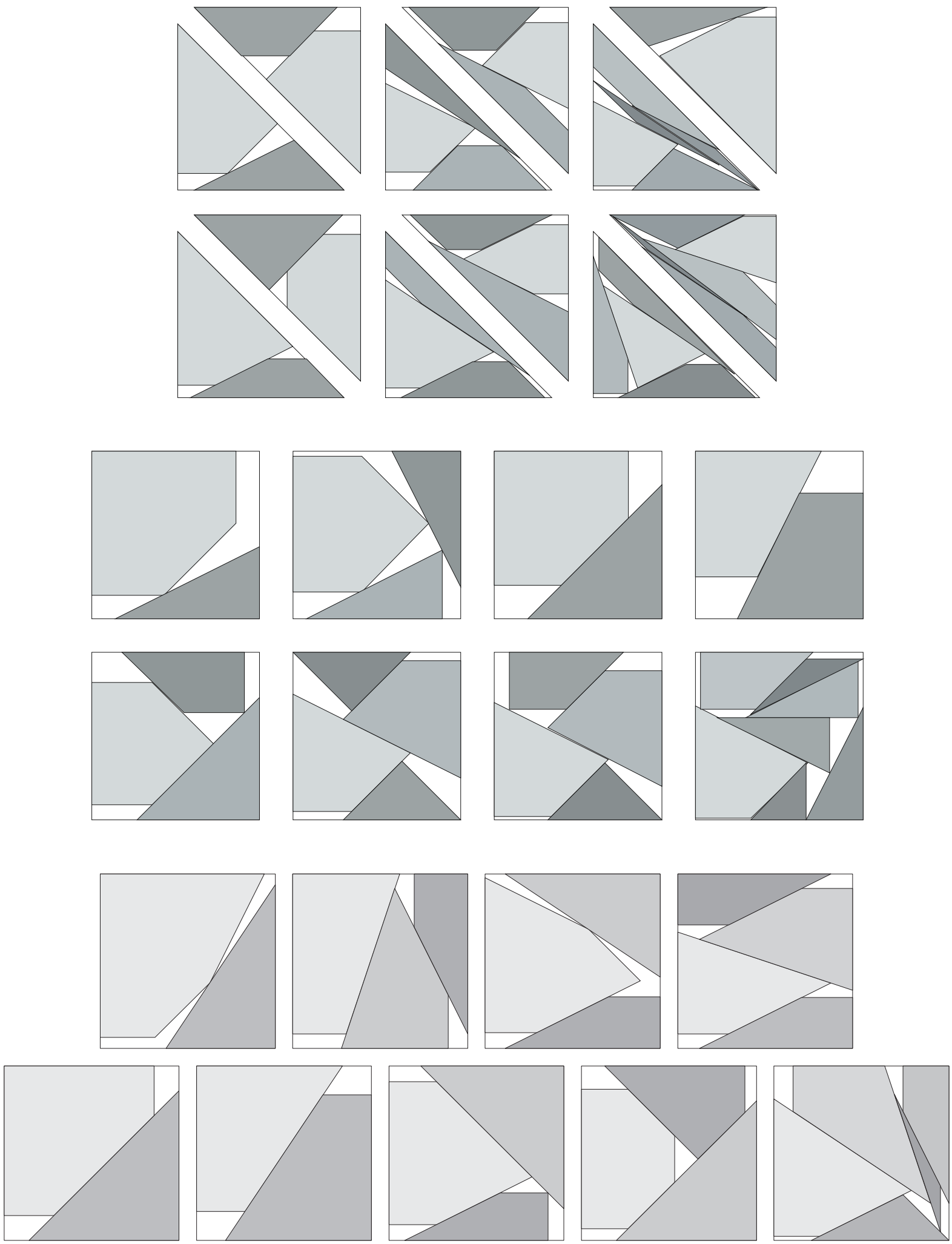

FIGURE 5.5. Top: Lattice polygon packings that yield packings of the standard simplex by $n$ triangles, where $10 \leq n \leq 14$ or $17 \leq n \leq 23$. Middle: Lattice polygon packings that yield packings of the square by $n$ triangles, where $9 \leq n \leq 16$. Bottom: Lattice polygon packings that yield packings of the square by $n$ triangles, where $19 \leq n \leq 27$. 
[Stein and Szabó 1994] S. K. Stein and S. Szabó, Algebra and tiling: homomorphisms in the service of geometry, Carus Mathematical Monographs 25, Mathematical Association of America, Washington, DC, 1994.

[Tokieda 1996] T. Tokieda, Null Sets of Symplectic Capacity, Ph.D. thesis, Princeton University, Princeton, NJ, 1996.
[Tokieda 1997] T. F. Tokieda, "Isotropic isotopy and symplectic null sets", Proc. Nat. Acad. Sci. U.S.A. 94:25 (1997), 13407-13408.

[Traynor 1995] L. Traynor, "Symplectic packing constructions", J. Differential Geom. 42:2 (1995), 411429.

[Xu 1994] G. Xu, "Curves in $\mathbb{P}^{2}$ and symplectic packings", Math. Ann. 299:4 (1994), 609-613.

F. Miller Maley, Center for Communications Research, IDA, Princeton, NJ 08540, United States (maley@idaccr.org)

Jean Mastrangeli, Mathematics Department, Immaculata College, Immaculata, PA 19345-0648, United States (jmastrangeli@immaculata.edu)

Lisa Traynor, Mathematics Department, Bryn Mawr College, Bryn Mawr, PA 19010, United States (ltraynor@brynmawr.edu)

Received August 19, 1999; accepted in revised form January 13, 2000 